\title{
Diagnostic performance of microRNAs in testicular germ cell tumors: a systematic review and meta-analysis
}

\author{
Xi-Yi Zhao ${ }^{1,}{ }^{*}$, Yu-Lu Gao ${ }^{1,2,}{ }^{*}$, Dan-Feng Li ${ }^{1}$, Hong-Chao Liu' ${ }^{1}$, Rui-Fang Zhu ${ }^{3}$, Chang-Tai Zhu ${ }^{1}$ \\ ${ }^{1}$ Department of Laboratory Medicine, Shanghai Jiao Tong University Affiliated Sixth People's Hospital, Shanghai 200233, \\ China \\ ${ }^{2}$ Department of Laboratory Medicine, Kunshan Affiliated Hospital of Nanjing University of Chinese Medicine, Kunshan \\ 215300, China \\ ${ }^{3}$ Editorial Department, First Hospital of Shanxi Medical University, Taiyuan, Shanxi, China \\ ${ }^{*}$ Equal contribution
}

Correspondence to: Rui-Fang Zhu, Chang-Tai Zhu; email: ruifang.zhu@sxmu.edu.cn; zct101@163.com,

https://orcid.org/0000-0002-5250-1937

Keywords: microRNAs, testicular germ cell tumors, diagnosis, biomarker, meta-analysis

Received: March 22, $2021 \quad$ Accepted: July 15, $2021 \quad$ Published: August 3, 2021

Copyright: (C) 2021 Zhao et al. This is an open access article distributed under the terms of the Creative Commons Attribution License (CC BY 3.0), which permits unrestricted use, distribution, and reproduction in any medium, provided the original author and source are credited.

\section{ABSTRACT}

The sensitivity (Sen) of classic biomarkers for the diagnosis of testicular germ cell tumors (TGCTs) is currently low. Previous studies have shown the diagnostic potential of microRNAs (miRNAs) for TGCTs; however, the results of these studies are inconsistent. Therefore, we conducted a systematic review and meta-analysis to evaluate their diagnostic value. PubMed, EMBASE, Cochrane Library, and Web of Science databases were systematically searched until September 30, 2020 and 18 trials from 11 studies involving 2,068 participants were included in this meta-analysis. Using a bivariate mixed-effects meta-analysis model, the pooled Sen, specificity (Spe), positive likelihood ratio (PLR), negative likelihood ratio (NLR), diagnostic odds ratio (DOR), and area under the curve (AUC) with 95\% confidence interval values of total miRNAs were $0.83(0.73-0.90)$, 0.95 (0.89-0.98), 15.79 (7.41-33.66), 0.18 (0.11-0.29), 87.13 (41.99-180.82), and $0.95(0.93-0.97)$, respectively; however, the observed values of single miR-371a-3p were $0.84(0.76-0.90), 0.95(0.91-0.98)$, 18.41 (9.69-34.97), $0.17(0.11-0.26), 111.56$ (47.72-260.80), and 0.97 (0.95-0.98), respectively. Subgroup analysis revealed that miRNAs that included miR-371a-3p showed higher predictive performance than those that did not $(P<0.05)$. This research identified that miR-371a-3p has a high diagnostic value for TGCTs, except teratoma.

\section{INTRODUCTION}

Testicular germ cell tumors (TGCTs) are diverse malignancies with different histological patterns originating from primitive germ cells [1]. Although TGCTs are relatively uncommon, accounting for $<1 \%$ of all tumors in men, this incidence has been increasing. Currently, TGCTs are the most common solid tumor observed in men aged 15 to 34 years [2]. The prognosis of patients with a TGCT has dramatically improved over the last 40 years because of the use of cisplatinbased therapy and other advancements in medical oncology [3]. However, the prognosis for TGCT is dependent on the clinical stage of cancer at the time of diagnosis, and survival rates of up to $97 \%$ have been achieved when patients are diagnosed in the early stages [4]. Therefore, early diagnosis and treatment of TGCTs can lead to improved outcomes.

Current guidelines from the Taussig Cancer Institute, Glickman Urological and Kidney Institute, and European Association of Urology recommend the use of human chorionic gonadotropin subunit $\beta, \alpha$-fetoprotein (AFP), and lactate dehydrogenase as serum markers for 
the clinical staging, treatment monitoring, and followup of patients with a TGCT [5-6]. However, the low sensitivity (Sen) and specificity (Spe) of these classical markers remains a major challenge in the diagnosis of TGCTs. Several studies have suggested that only $50 \%$ of seminoma and $75 \%$ of non-seminoma express one of these three markers [7]. Additionally, these classical markers are also expressed in many other diseases, such as hepatocellular carcinoma, pancreatic cancer, gastric cancer, viral hepatitis and others, suggesting the low Spe for TGCTs [8]. Hence, new biomarkers with greater Sen and Spe for TGCTs must be identified.

MicroRNAs (miRNAs) are small non-coding RNAs (19-22 nucleotides in length) that are involved in the regulation of mRNA transcription and translation [9]. Specific sequences of miRNAs may have significant effects on the transcriptional regulation of carcinogenesis [10]. Recent studies have shown the clinical potential of miR-371-373 and miR-302/367 clusters in the diagnosis, monitoring, and follow-up of patients with a TGCT. The miR-371a-3p, miR-373-3p, miR$367-3 p$ and some other miRNAs exhibited higher levels in TGCT patients. Furthermore, these miRNAs showed significantly improved diagnostic value for the diagnosis of TGCTs with Sen of 75-100\% compared with classic biomarkers with Sen of $50-60 \%$, and the Spe of miRNAs could also be $80-100 \%$ in different studies [11-12]. However, the results of these studies on different miRNAs are inconsistent and need to be synthesized for a better understanding of how the expression of these miRNAs is associated with TGCTs. Thus, we conducted a systematic review and metaanalysis of clinical trials to evaluate the role of miRNAs in TGCT diagnosis.

\section{MATERIALS AND METHODS}

\section{Protocol}

This systematic review and meta-analysis was conducted in accordance with the Cochrane Handbook for Systematic Reviews of Diagnostic Test Accuracy and Standards formulated in Preferred Reporting Items for Systematic Reviews and Meta-analyses (PRISMA) [13].

\section{Literature search strategy}

Two researchers independently searched the PubMed, EMBASE, Cochrane Library, and Web of Science databases using the keywords for TGCTs and miRNAs in all articles published until September 30, 2020. The literature retrieval search terms were as follows: ("MicroRNA" or "miRNAs" or "RNA, Micro" or "Primary miRNA" or "miRs" or "microRNAs") and ("Testicular Neoplasms" or "Testicular Tumors" or
"Testicular Cancers" or "Testis Neoplasms" or "Testis Cancers" or "Testis Tumors" or "Germinomas" or "Testicular Germ Cell Tumors" or "Testicular Germ Cell Cancers"). In addition, the references listed in the identified articles were scrutinized for relevant research. The search strategy for PubMed is described in Supplementary Table 1.

\section{Inclusion and exclusion criteria}

The inclusion criteria were as follows: (1) the study included patients with a TGCT and those with nonmalignant testicular diseases (NMTDs) or healthy males as controls; (2) samples of miRNAs were isolated from plasma, serum, or tissue; (3) histopathological examination of patients was conducted and appropriate controls were used as reference standards; and (4) sufficient data were available to obtain true positive (TP), false positive (FP), false negative (FN), and true negative (TN) values. The exclusion criteria were as follows: (1) duplicate publications; (2) incomplete or unavailable data; (3) letters, reviews, editorials, or case reports; and (4) a sample size of less than 10.

\section{Data extraction and quality assessment}

Two reviewers (Zhao and Liu) independently extracted data from the included studies by filling the standardized forms. Disagreements were resolved by consulting the third reviewer (Zhu). The following data were extracted from the included studies: name of the first author, year of publication, country, general characteristics of participants, source of the samples, and results of diagnostic tests. TP, FP, FN, and TN were directly extracted from the original publications or indirectly calculated based on the sample size, Sen, and Spe data. Quality Assessment of Diagnostic Accuracy Studies-2 (QUADAS-2) was used to assess the quality of the included studies. The QUADAS-2 score system considers the selection index of patients, index tests, reference standards, flow and timing to judge the bias risk, and applicability of diagnostic studies. All included studies were assessed on the basis of seven items (four items of the risk of bias and three items of applicability). The quality of each item was classified as high, undefined, or low risk and the quality of each included study was classified as low, medium, or high quality [14].

\section{Statistical analyses}

Statistical analyses were performed using Stata 13.1 (Stata Corp, College Station, TX, USA), GraphPad Prism 6.02 (GraphPad Software, San Diego, CA, USA), and Review Manager 5.2 (Cochrane Collaboration, Oxford, UK). 
Diagnostic meta-analyses of total miRNAs; miRNAs, including miR-371a-3p; and miRNAs, excluding miR-371a-3p; were conducted. The pooled Sen, Spe, positive likelihood ratio (PLR), negative likelihood ratio (NLR), diagnostic odds ratio (DOR), and area under the curve (AUC) with 95\% confidence interval (CI) were calculated based on a bivariate mixedeffects meta-analysis model [15]. A $P$ value less than 0.05 was considered statistically significant. The heterogeneity among studies was assessed using the $I^{2}$ test, and if $I^{2}$ was $0-25 \%, 26-50 \%, 51-75 \%$, or > $75 \%$, the degree of heterogeneity among the included studies was considered to be not significantly, mildly, moderately, or highly heterogeneous, respectively [16-17].

Spearman's correlation coefficient between Sen and 1-Spe was used to quantitatively assess the threshold effect [18]. Results from the receiver operating characteristic space (ROC plane) were analyzed to qualitatively assess the threshold effect visually [19].

To identify factors that influenced Sen and Spe, metaregression and subgroup analyses were performed using the following independent variables: design type (retrospective or prospective), controls (patients with NMTDs included or healthy males alone), specimen type (serum or other samples), miRNA type (miR-371a$3 p$ included or excluded), and miRNA number (single or multiple miRNAs) [20]. In addition, a random-effects model using the method of DerSimonian and Laird, with the command "metaprop" in the Stata software was adopted to perform comparisons between different subgroups. To observe the independent diagnostic value of miR-371a-3p, a stratified analysis of miRNAs (single miR-371a-3p or multiple miRNAs with miR-371a-3p) was conducted. Sen analyses were conducted for the diagnostic parameters involving Sen and Spe through stepwise exclusion to determine whether the metaanalysis results were robust [21].

Publication bias was determined using Deeks' funnel plot with DOR as the dependent variable and the reciprocal of the square root of the effective sample size as an independent variable. A $P$ value less than 0.05 was considered to indicate statistically significant difference [22-23].

Likelihood ratio scattergrams were used to evaluate the clinical utility of miRNAs as diagnostic biomarkers in patients with TGCTs. The results were classified into four quadrants based on the summary values of PLR and NLR. Specifically, PLR $>10$ and NLR $<0.1$ indicated that the target biomarker reached the laboratory diagnostic standard for confirmation and exclusion, respectively [24].
To evaluate the external validity of miRNAs for TGCTs, diagnostic probability line charts and Fagan's plots were constructed based on pre- and post-test probabilities [25, 26]. The TGCT pre-test probability for diagnostic probability line charts was estimated to be between $0 \%$ and $50 \%$, and post-test positive (negative) probability was calculated correspondingly. Fagan plots were constructed based on a pre-test probability of $20 \%$.

\section{Evidence quality assessments}

The GRADEpro Guideline Development Tool (GRADEpro GDT) online software was used to assess the quality of evidence for the main outcomes [27]. The GRADE criteria for the diagnostic test include risk of bias, indirectness, inconsistency, imprecision, and publication bias. The certainty of evidence can be classified into the following four grades: (1) high, meaning further research is very unlikely to change confidence in the estimate of effect size and direction; (2) moderate, meaning further research may change the estimate or affect confidence in it; (3) low, meaning further research is very likely to change the estimate and affect confidence in it; and (4) very low, meaning the effect cannot be estimated accurately [28].

\section{RESULTS}

\section{Literature search}

The initial literature search yielded 533 potentially relevant studies. After removing 315 duplicate articles, we screened 218 articles, of which 49 were read. We finally selected 11 studies on 18 trials that met the eligibility criteria for further data extraction and analysis [29-39]. The literature search process is presented as a flowchart in Figure 1.

\section{Study characteristics and quality assessment}

The 11 selected studies involving 18 trials included 1,321 patients and 747 controls. The controls of the three studies were healthy males [33, 35, 39], whereas the others included patients with NMTDs. Of the 11 studies, two adopted a prospective design [29, 30], whereas others adopted a retrospective design. Two studies used tissue as specimens [31, 39], whereas the others employed serum. All studies used quantitative real-time reverse transcription PCR to detect miRNA expression. The target miRNAs of 13 of the 18 trials included miR-371a-3p and 4 of the 18 trials used multiple miRNAs as biomarkers (Table 1). A Cochrane bias graph was constructed using the QUADAS-2 tool to evaluate the quality of each included study. Of the 11 studies, 10 involving 17 trials were of high quality [2938 ] and only one study was of low quality because of 
inappropriate patient selection (consecutive sample selection and avoidance of inappropriate exclusions were lacking) [39]. All included studies were doubleblinded and used histopathological examination as the reference standard. Eight studies indicated the cut-off values [29-34, 36, 38], whereas the other three did not $[35,37,39]$. The overall quality of the included studies was high (Figure 2).

\section{Meta-analysis results of total miRNAs for TGCTs}

The pooled Sen, Spe, PLR, NLR, DOR, and AUC values of total miRNAs were $0.83(0.73-0.90 ; 95 \% \mathrm{CI}), 0.95$
(0.89-0.98), 15.79 (7.41-33.66), $0.18(0.11-0.29), 87.13$ (41.99-180.82), and 0.95 (0.93-0.97), respectively (Figure 3 ). The $I^{2}$ values for Sen, Spe, PLR, and NLR of these miRNAs were $93.83 \%, 90.20 \%, 87.78 \%$, and $96.04 \%$, respectively.

\section{Threshold effect}

The Spearman's correlation coefficients of total miRNAs and single miR-371a-3p were $0.438(P=0.069, P>0.05)$ and $0.233(P=0.546, P>0.05)$, respectively. Moreover, the ROC plane of both the total miRNAs and single miR-371a-3p did not show a "shoulder-arm" shape

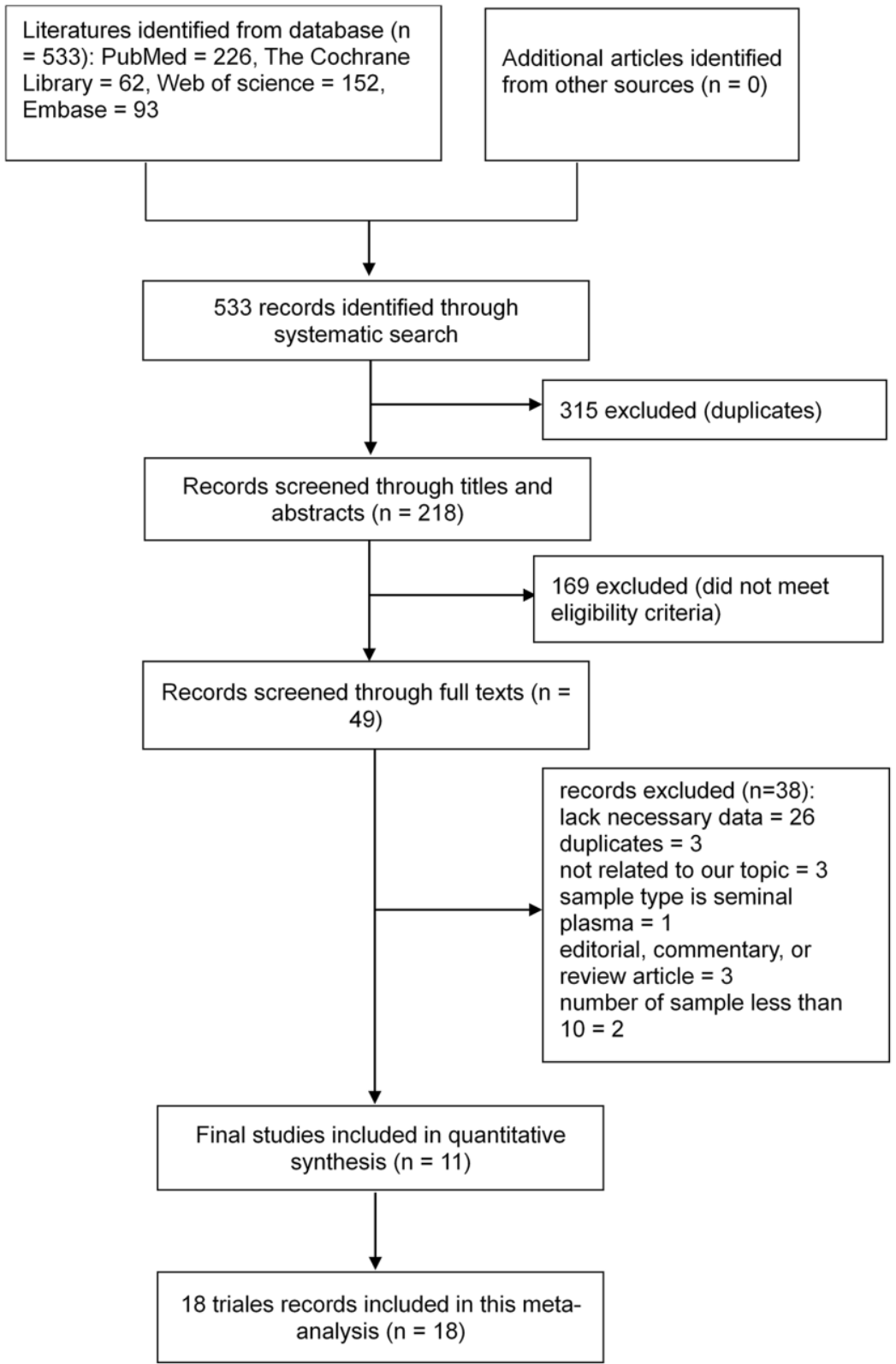

Figure 1. Flowchart of the study selection process. Based on the inclusion and exclusion criteria, 533 records were identified and 218 records with associated abstracts were reviewed. Of these, 49 records were selected for full review, and 11 studies on 18 trials met the eligibility criteria for further data extraction and analysis. 
Table 1. Characters of included studies.

\begin{tabular}{|c|c|c|c|c|c|c|c|c|c|c|c|c|c|c|c|c|}
\hline \multirow{2}{*}{$\begin{array}{l}\text { References } \\
\text { included }\end{array}$} & \multirow{2}{*}{ Country } & \multirow{2}{*}{ Age (year) } & \multirow{2}{*}{ Specimen } & \multirow{2}{*}{ Design } & \multirow{2}{*}{$\begin{array}{c}\text { Target } \\
\text { microRNAs }\end{array}$} & \multirow{2}{*}{$\begin{array}{l}\text { Cut-off } \\
\text { value }\end{array}$} & \multicolumn{4}{|c|}{ Case composition } & \multicolumn{2}{|c|}{ Control composition } & \multicolumn{4}{|c|}{ Result } \\
\hline & & & & & & & GCNIS & SE & NS & Total & Control & Total & $\mathbf{T P}$ & FP & FN & TN \\
\hline \multirow{4}{*}{$\begin{array}{l}\text { Morup N } \\
2020 \text { [29] }\end{array}$} & \multirow{4}{*}{ Denmark } & \multirow{4}{*}{ NR } & \multirow{4}{*}{ Serum } & \multirow{4}{*}{ Prospective } & miR-367-3p & $\mathrm{Ct}=40$ & 0 & 17 & 23 & 40 & NMTD & 22 & 8 & 0 & 32 & 22 \\
\hline & & & & & $\operatorname{miR}-371 a-3 p$ & $\mathrm{Ct}=40$ & 0 & 17 & 23 & 40 & NMTD & 22 & 27 & 0 & 13 & 22 \\
\hline & & & & & $\operatorname{miR}-372-3 p$ & $\mathrm{Ct}=40$ & 0 & 17 & 23 & 40 & NMTD & 22 & 22 & 0 & 18 & 22 \\
\hline & & & & & $\operatorname{miR}-373-3 p$ & $\mathrm{Ct}=40$ & 0 & 17 & 23 & 40 & NMTD & 22 & 25 & 0 & 15 & 22 \\
\hline $\begin{array}{c}\text { Dieckmann } \\
\text { KP 2019 [30] } \\
\end{array}$ & $\begin{array}{c}\text { Germany } \\
\text { et al. }\end{array}$ & $16.0-69.0$ & Serum & Prospective & $\operatorname{miR}-371 a-3 p$ & $\mathrm{RQ}=5$ & 0 & 323 & 199 & 522 & HM + NMTD & 258 & 479 & 10 & 43 & 248 \\
\hline \multirow{2}{*}{$\begin{array}{c}\text { Vilela- } \\
\text { SalgueiroB } \\
2018[31]\end{array}$} & \multirow{2}{*}{ Portugal } & $13.0-52.0$ & Tissue & Retrospective & miR-371a-3p & $\mathrm{RE}=0.0875$ & 0 & 68 & 35 & 103 & NMTD & 15 & 95 & 1 & 8 & 14 \\
\hline & & $1.0-35.0^{\$}$ & Tissue & Retrospective & miR-371a-3p & $\mathrm{RE}=0.0875$ & 0 & 0 & 16 & 16 & NMTD & 15 & 11 & 3 & 5 & 12 \\
\hline $\begin{array}{l}\text { Radtke A } \\
2017 \text { [32] }\end{array}$ & Germany & $35.3 \pm 8.8$ & Serum & Retrospective & $\operatorname{miR}-371 a-3 p$ & $R Q=5$ & 27 & 0 & 0 & 27 & HM + NMTD & 20 & 14 & 1 & 13 & 19 \\
\hline $\begin{array}{l}\text { Pelloni M } \\
2017 \text { [33] }\end{array}$ & Italy & NR & Serum & Retrospective & $\operatorname{miR}-371 a-3 p$ & $R Q=5$ & 0 & 23 & 5 & 28 & $\mathrm{HM}$ & 28 & 25 & 0 & 3 & 28 \\
\hline $\begin{array}{c}\text { Dieckmann } \\
\text { KP } 2017 \text { [34] } \\
\end{array}$ & Germany & $18.0-60.0$ & Serum & Retrospective & $\operatorname{miR}-371 a-3 p$ & $\mathrm{Ct}=40$ & NR & NR & NR & 150 & HM + NMTD & 106 & 133 & 7 & 17 & 99 \\
\hline \multirow{4}{*}{$\begin{array}{c}\text { Van } \\
\text { Agthoven T } \\
2016[35]\end{array}$} & \multirow{4}{*}{ Holland } & \multirow{4}{*}{$12.0-81.0$} & \multirow{4}{*}{ Serum } & \multirow{4}{*}{ Retrospective } & miR-371a-3p & NR & 0 & 128 & 110 & 238 & $\mathrm{HM}$ & 104 & 212 & 10 & 26 & 94 \\
\hline & & & & & miR-373-3p & NR & 0 & 128 & 110 & 238 & $\mathrm{HM}$ & 104 & 167 & 11 & 71 & 93 \\
\hline & & & & & miR-367-3p & NR & 0 & 128 & 110 & 238 & $\mathrm{HM}$ & 104 & 188 & 16 & 50 & 88 \\
\hline & & & & & $\begin{array}{c}\text { miR-371a- } \\
\text { 3p/miR-373- } \\
\text { 3p/miR-367-3p }\end{array}$ & NR & 0 & 128 & 110 & 238 & $\mathrm{HM}$ & 104 & 219 & 9 & 19 & 95 \\
\hline $\begin{array}{c}\text { Rijlaarsdam } \\
\text { MA 2015 } \\
{[36]} \\
\end{array}$ & Holland & NR & Serum & Retrospective & $\begin{array}{c}\text { miR-371- } \\
\text { 373/miR-511 } \\
\text { et al. }{ }^{\&} \\
\end{array}$ & $\mathrm{Ct}=40$ & 0 & 14 & 10 & 24 & HM + NMTD & 11 & 22 & 5 & 2 & 6 \\
\hline $\begin{array}{c}\text { Syring I } 2015 \\
{[37]} \\
\end{array}$ & Germany & NR & Serum & Retrospective & miR-371a-3p & NR & NR & NR & NR & 59 & HM + NMTD & 101 & 50 & 1 & 9 & 100 \\
\hline $\begin{array}{l}\text { Gillis AJ } \\
2013[38]\end{array}$ & $\begin{array}{l}\text { Holland } \\
\text { et al. }\end{array}$ & NR & Serum & Retrospective & $\begin{array}{l}\text { miR-371a- } \\
\text { 3p/miR-367 }\end{array}$ & $\begin{array}{c}\mathrm{CT}= \\
15.62 / 12.48^{\S}\end{array}$ & 0 & NR & NR & 80 & HM + NMTD & 59 & 79 & 31 & 1 & 28 \\
\hline $\begin{array}{c}\text { Palmer R.D } \\
2010 \text { [39] }\end{array}$ & UK & NR & Tissue & Retrospective & $\begin{array}{c}\mathrm{miR}-371-373 \\
\text { cluster/miR-302 } \\
\text { cluster } \\
\end{array}$ & NR & 0 & 13 & 21 & 34 & $\mathrm{HM}$ & 8 & 33 & 0 & 1 & 8 \\
\hline
\end{tabular}

Abbreviations: NR: not reported; GCNIS: germ cell neoplasia in situ; SE: Seminoma; NS: Nonseminoma; NMTD: non-malignant tumor disease; HM: healthy male; miR: microRNA; TP: true positive; FP: false positive; FN: false negative; TN: true negative; Ct: cycle threshold; RQ: relative quantity; RE: relative expression level. "Germany/Austria/Switzerland/ Italy; "Holland/Germany/UK; \$ one study on two trials with different age and the same other factors; ${ }^{8} 750$ microRNAs included miR-371-373/miR-511 et al.; ${ }^{\circledR}$ Cut-off values of miR$371 a-3 p$ and miR-367 are 15.62 and 12.48 , respectively.

(Supplementary Figure 1). Hence, a threshold effect did not exist.

\section{Meta-regression, subgroup, and stratified analyses}

Meta-regression analysis showed that the inclusion of miR-371a-3p might be a source of heterogeneity for Sen $(P<0.05)$ and whether biomarkers of single or multiple miRNAs were used might be a source of heterogeneity for Spe $(P<0.05$; Supplementary Table 2$)$.

Higher diagnostic accuracy in studies of miRNAs with miR-371a-3p than those without miR-371a-3p was observed with a Sen value of $0.89(0.82-0.93,95 \% \mathrm{CI})$ versus $0.58(0.38-0.75)$ and AUC value of $0.96(0.94$ $0.97)$ versus $0.86(0.83-0.89 ; P=0.001)$. Compared with the studies that involved a single miRNA, those that involved multiple miRNAs showed a higher Sen value of $0.95(0.91-1.00)$ versus $0.74(0.67-0.87, P=$ 0.000). Prospective studies had a higher Spe of 0.97 $(0.95-0.99)$ versus $0.89(0.83-0.94, P=0.003)$ in retrospective studies. Moreover, the subgroup of tissue samples showed a higher Sen of $0.92(0.84-1.00)$ compared to $0.77(0.71-0.84, P=0.006)$ in the subgroup of serum samples. However, other diagnostic parameters were not significantly different between subgroups. The details are listed in Table 2.

Stratified analysis revealed that single miR-371a-3p had a Sen of $0.84(0.76-0.90)$, which was lower than that of $0.95(0.91-1.00, P=0.005)$ observed in multiple miRNAs with miR-371a-3p. However, other diagnostic parameters showed that there were no significant differences between single miR-371a-3p and multiple miRNAs with miR-371a-3p, such as AUC of $0.97(0.95-0.98)$ versus $0.96(0.94-0.98, P=$ 0.694) (Table 3).

\section{Sensitivity analysis and publication bias}

Sen analysis revealed no significant change in Sen or Spe (Supplementary Figure 2). Studies of total miRNAs and 
single miR-371a-3p showed no publication bias (Deeks' test, $P=0.29$, and $P=0.22$, respectively; Supplementary Figure 3).

\section{Clinical utility}

The likelihood ratio scattergram showed that studies with total miRNAs and single miR-371a-3p were located in the upper right quadrant, indicating that they reached the laboratory diagnostic standard for confirmation but not exclusion (Additional files 6: Supplementary Figure 4).
The diagnostic probability line charts showed that both the total miRNAs and single miR-371a-3p exhibited an increase in post-test positive probability and a decrease in post-test negative probability compared to the TGCT pretest probability. Although they showed similar values in the negative test, single miR-371a-3p showed higher values than total miRNAs in the positive test. Fagan's plots also confirmed that the post-test positive probability of total miRNAs $(80 \%)$ was lower than that of single miR371a-3p (82\%), whereas the post-test negative probability did not differ between total miRNAs and single miR-371a$3 p$ (4\% versus 4\%; Supplementary Figure 5).

A

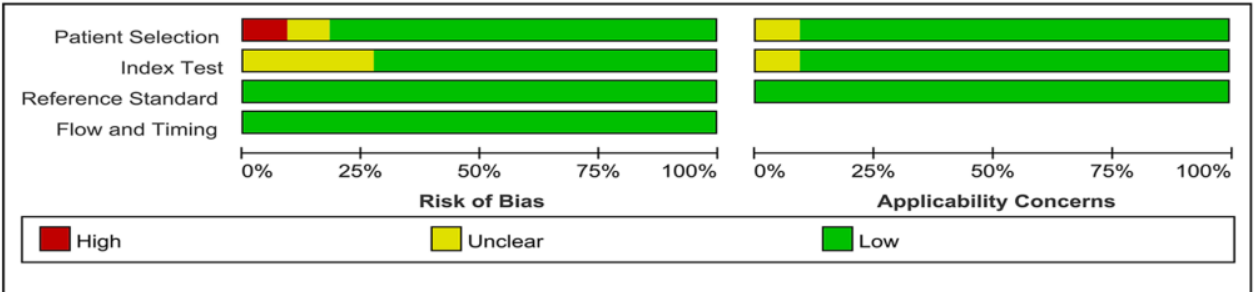

B

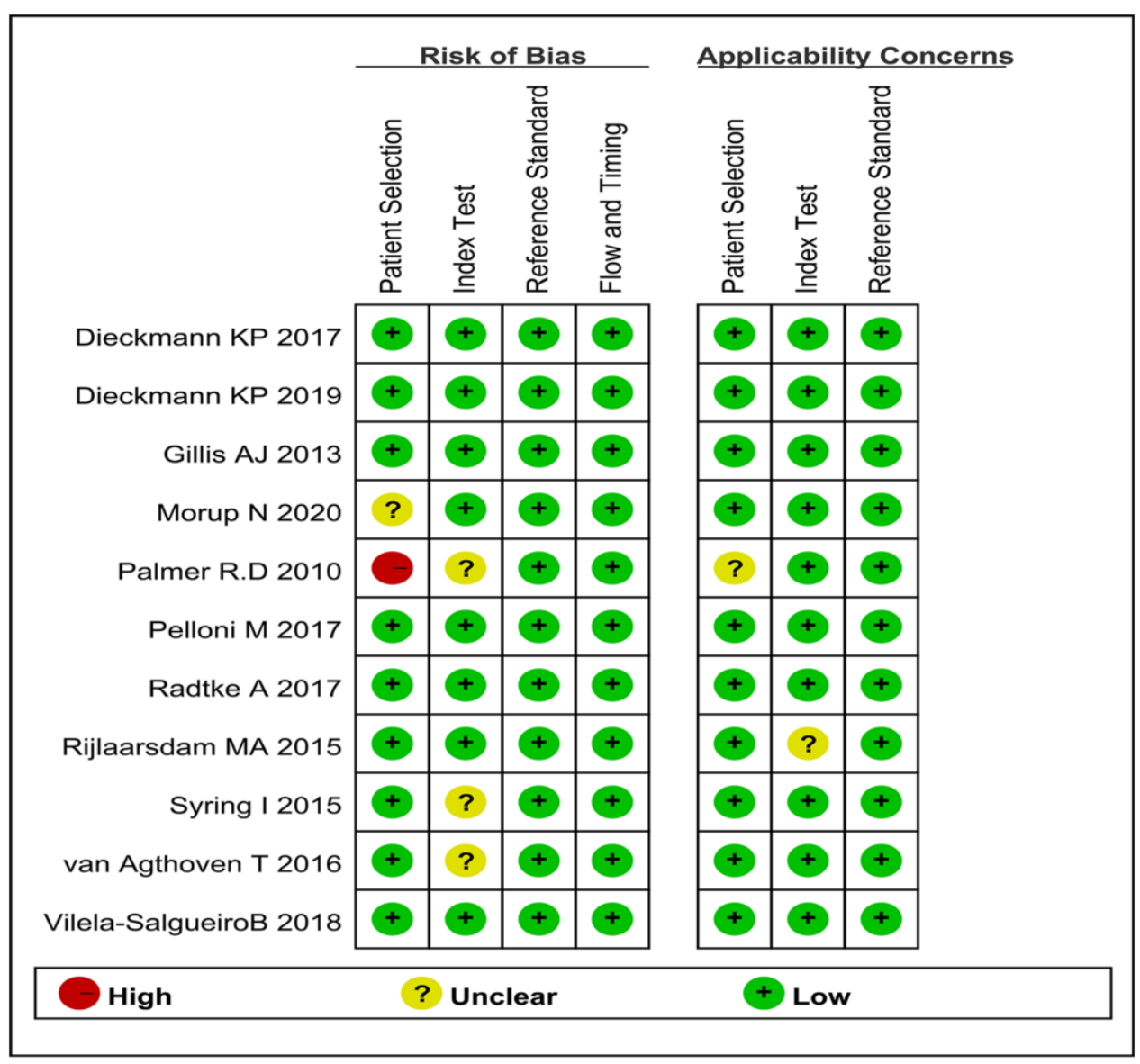

Figure 2. Bias risks and applicability concerns: qualification. (A) Risk of bias and applicability concerns graph (review authors' judgments about each domain presented as percentages across 11 studies on 18 trials); (B) Risk of bias and applicability concerns summary (review authors' judgments about each domain for each included study). 


\section{Evidence quality assessment}

The quality of evidence for microRNAs included miR-371a-3p, microRNAs not included miR-371a-3p, multiple microRNAs with miR-371a-3p, and single miR-371a-3p as biomarkers for TGCT was graded as moderate because of the large inconsistencies among the included studies (GRADE) (Table 4).

A

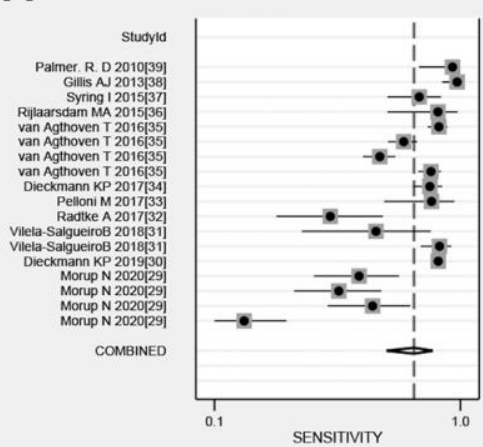

SENSITIVITY (95\% CI)

$0.97[0.85-1.00]$
0.99

$0.99[0.93-1.00$
0.85
$0.92 .73-0.93]$

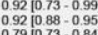

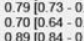

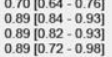

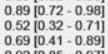

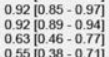

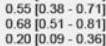

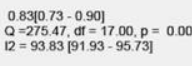

\section{DISCUSSION}

The incidence of TGCTs has increased over the last few decades, and TGCT is now the most common solid tumor among men aged 15 to 34 years. However, the classic biomarkers recommended by current guidelines consisting of human chorionic gonadotropin subunit $\beta$, $\mathrm{AFP}$, and lactate dehydrogenase have relatively limited

\section{B}

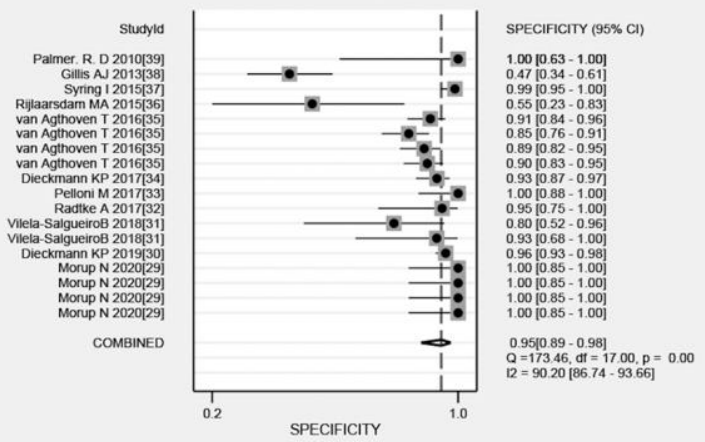

D
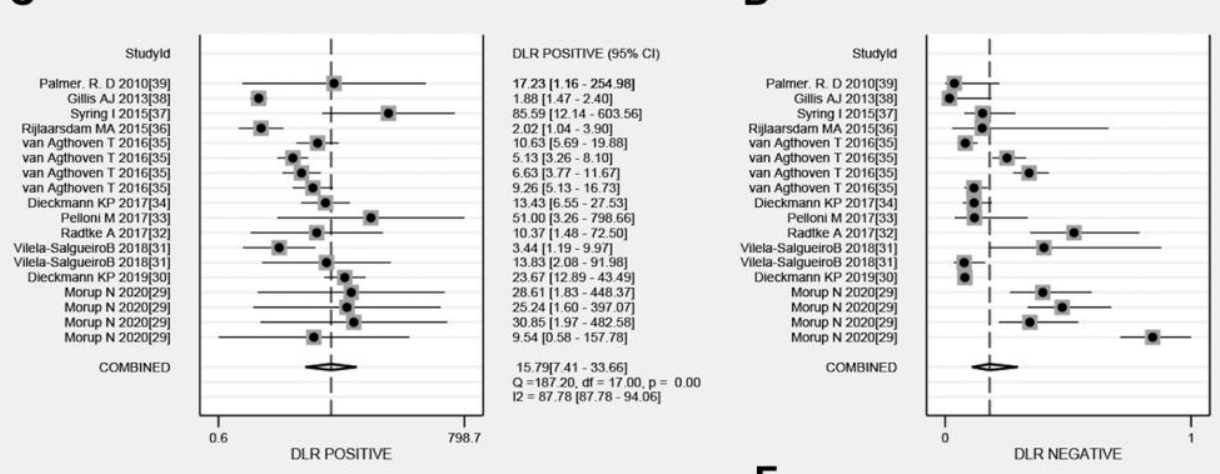

DLR NEGATIVE (95\% Cl)

$0.05[0.01-0.22]$
$0.03[0.01-0.19]$

$0.15[0.08-0.28]$
$0.15[0.04-0.64]$

0.15
0.09
$0.02506-0.14$
$0.019-0.32$

$0.25[0.19-0.32]$
$0.33[0.27-0.41]$
$0.12008-0.17$

$0.12[0.08-0.19]$

$0.51[0.34-0.76$

0.39
0.08
$0.0904-0.16$
0.09

$0.39[0.26-0.57]$
$0.46[0.33-0.65]$

$0.46[033-0.65]$
$0.34[022-0.52]$
$0.81[0.69-0.96]$

$0.18[0.11-0.29]$
$Q=428.92$, df $=17.00, p=0.00$

$\mathbf{F}$

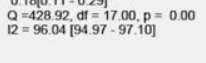

E

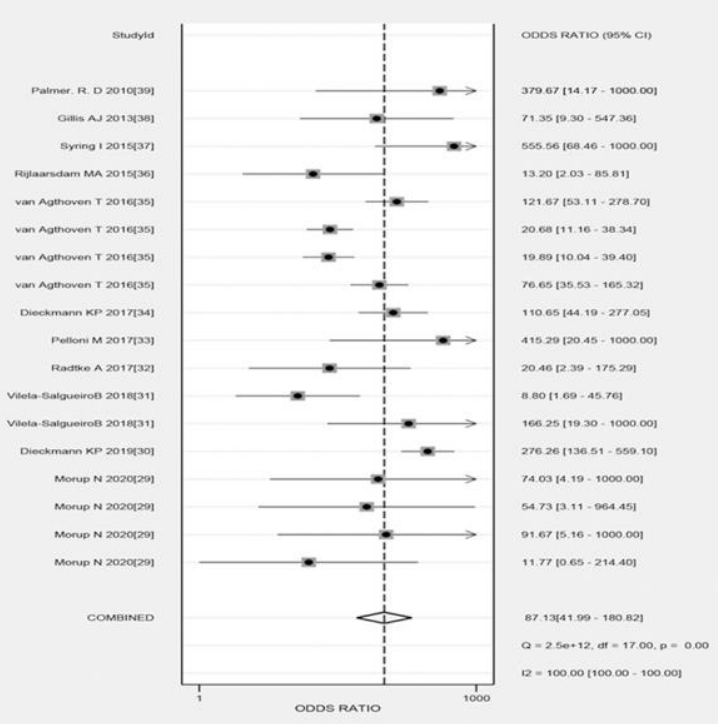



Figure 3. Forest plots of total microRNAs. (A) Sen of total microRNAs in TGCT; (B) Spe of total microRNAs in TGCT; (C) PLR of total microRNAs in TGCT; (D) NLR of total microRNAs in TGCT; (E) DOR of total microRNAs in TGCT; (F) AUC of total microRNAs in TGCT. 
Table 2. Sensitivity and specificity subgroup analyses.

\begin{tabular}{|c|c|c|c|c|}
\hline \multirow{2}{*}{ Subgroups } & Sensitivity & \multirow{2}{*}{$\boldsymbol{P}$} & Specificity & \multirow{2}{*}{$\boldsymbol{P}$} \\
\hline & $(95 \% \mathrm{CI})$ & & $(95 \% \mathrm{CI})$ & \\
\hline \multicolumn{5}{|c|}{ Comparison group: Design type of prospective or retrospective } \\
\hline Prospective $(n=5)$ & $0.60(0.30,0.89)$ & 0.082 & $0.97(0.95,0.99)$ & 0.005 \\
\hline Retrospective $(n=13)$ & $0.86(0.81,0.92)$ & & $0.89(0.83,0.94)$ & \\
\hline \multicolumn{5}{|c|}{ Comparison group: Specimen type of serum or tissue } \\
\hline Serum $(n=15)$ & $0.77(0.71,0.84)$ & 0.006 & $0.92(0.89,0.96)$ & 0.855 \\
\hline Tissue $(n=3)$ & $0.92(0.84,1.00)$ & & $0.91(0.82,1.00)$ & \\
\hline \multicolumn{5}{|c|}{ Comparison group: Controls of NMTD included or not } \\
\hline HM along $(n=6)$ & $0.86(0.79,0.93)$ & 0.051 & $0.91(0.87,0.95)$ & 0.658 \\
\hline NMTD included $(n=12)$ & $0.75(0.67,0.83)$ & & $0.92(0.88,0.97)$ & \\
\hline \multicolumn{5}{|c|}{ Comparison group: microRNAs included miR-371a-3p or not } \\
\hline Included $(n=13)$ & $0.89(0.86,0.93)$ & 0.001 & $0.91(0.87,0.95)$ & 0.674 \\
\hline Not included $(n=5)$ & $0.58(0.40,0.75)$ & & $0.94(0.88,0.99)$ & \\
\hline \multicolumn{5}{|c|}{ Comparison group: Single microRNA or multiple microRNAs } \\
\hline Single $(n=14)$ & $0.74(0.67,0.81)$ & 0.000 & $0.95(0.93,0.97)$ & 0.121 \\
\hline Multiple $(n=4)$ & $0.95(0.91,1.00)$ & & $0.73(0.48,0.98)$ & \\
\hline
\end{tabular}

Abbreviations: $\mathrm{Cl}$ : confidence interval; Sen: sensitivity; Spe: specificity; HM: healthy males; NMTD: non-malignant testicular diseases.

Table 3. Stratified analysis of microRNAs included miR-371a-3p.

\begin{tabular}{|c|c|c|c|c|c|c|}
\hline \multirow{2}{*}{ Subgroups } & \multicolumn{6}{|c|}{ Median $(95 \%$ CI $)$} \\
\hline & Sen & Spe & PLR & NLR & DOR & AUC \\
\hline \multicolumn{7}{|c|}{ Comparison group: single miR-371a-3p or multiple microRNAs with miR-371a-3p } \\
\hline $\begin{array}{l}\text { Single } \\
(n=9)\end{array}$ & $\begin{array}{c}0.84 \\
(0.76,0.90)\end{array}$ & $\begin{array}{c}0.95 \\
(0.91,0.98)\end{array}$ & $\begin{array}{c}18.41 \\
(9.69,34.97)\end{array}$ & $\begin{array}{c}0.17 \\
(0.11,0.26)\end{array}$ & $\begin{array}{c}111.56 \\
(47.72,260.80)\end{array}$ & $\begin{array}{c}0.97 \\
(0.95,0.98)\end{array}$ \\
\hline $\begin{array}{c}\text { Multiple } \\
(n=4)\end{array}$ & $\begin{array}{c}0.95 \\
(0.91,1.00)\end{array}$ & $\begin{array}{c}0.73 \\
(0.48,0.98)\end{array}$ & $\begin{array}{c}4.12 \\
(1.68,10.11)\end{array}$ & $\begin{array}{c}0.06 \\
(0.03,0.11)\end{array}$ & $\begin{array}{c}69.43 \\
(26.63,181.01)\end{array}$ & $\begin{array}{c}0.96 \\
(0.94,0.98)\end{array}$ \\
\hline$P$ value & 0.005 & 0.128 & 0.078 & 0.051 & 0.588 & 0.694 \\
\hline
\end{tabular}

Abbreviations: miR: microRNA; $\mathrm{Cl}$ : confidence interval; Sen: sensitivity; Spe: specificity; PLR: positive likelihood ratio; NLR: negative likelihood ratio; DOR: diagnostic odds ratio; AUC: area under the curve.

diagnostic value, especially because of low overall Sen and Spe. Previous studies have noted the importance of miRNAs, especially miR-371-373 and miR-302/367 clusters, in TGCT diagnosis. Nevertheless, quantitative analyses of miRNA biomarkers have shown conflicting or inconsistent results, owing to the variations in study designs and target miRNAs. Therefore, we conducted a comprehensive meta-analysis to explore the diagnostic and clinical applications of miRNAs as novel biomarkers for TGCTs.

Our meta-analysis of 11 studies involving 18 trials comprised 1,321 patients with TGCTs and germ cell neoplasia in situ (GCNIS), SE, or non-SE (NS), and 747 controls (patients with NMTDs or healthy males). The total miRNAs presented a pooled diagnostic Sen of $83 \%$, Spe of $95 \%$, and AUC of 0.95 . The results of the stratified analysis showed that single miR-371a-3p could be the best miRNA marker with a pooled diagnostic Sen of $84 \%$, Spe of $95 \%$, and AUC of 0.97 . As for classic biomarkers, several studies have suggested that only $50 \%$ of SE and $75 \%$ of NS can express one of three classic markers [7], while they are also expressed in many other diseases, such as hepatocellular carcinoma, pancreatic cancer, gastric cancer, viral hepatitis, and others [8], suggesting a lower diagnostic value for TGCT 
Table 4. Evaluation of GRADE in the diagnostic performance of microRNAs in testicular germ cell tumor.

\begin{tabular}{|c|c|c|c|c|c|c|c|c|}
\hline \multirow[b]{2}{*}{ Outcome } & \multirow{2}{*}{$\begin{array}{l}\text { No. of studies } \\
\text { (No. of patients) }\end{array}$} & \multirow[b]{2}{*}{ Study design } & \multicolumn{5}{|c|}{ Factors that may decrease certainty of evidence } & \multirow{2}{*}{$\begin{array}{c}\text { Test accuracy } \\
\text { CoE }\end{array}$} \\
\hline & & & Risk of bias & Indirectness & Inconsistency & Imprecision & $\begin{array}{l}\text { Publication } \\
\text { bias }\end{array}$ & \\
\hline \multicolumn{9}{|c|}{ microRNAs included miR-371a-3p } \\
\hline \multicolumn{2}{|c|}{ Sensitivity } & \multicolumn{2}{|c|}{0.89 (95\% CI: 0.86 to 0.93$)$} & \multicolumn{2}{|c|}{ Specificity } & \multicolumn{3}{|c|}{$0.91(95 \%$ CI: 0.87 to 0.95$)$} \\
\hline $\begin{array}{l}\text { TP } \\
\text { FN }\end{array}$ & $\begin{array}{l}13 \text { studies [29-39] } \\
\text { (1321 patients) }\end{array}$ & $\begin{array}{l}\text { cohort and case- } \\
\text { control type studies }\end{array}$ & not serious & not serious & serious $^{\mathrm{a}}$ & not serious & none & $\begin{array}{c}\oplus \oplus \oplus \bigcirc \\
\text { MODERATE }\end{array}$ \\
\hline $\begin{array}{l}\text { TN } \\
\text { FP }\end{array}$ & $\begin{array}{l}13 \text { studies [29-39] } \\
\text { (669 patients) }\end{array}$ & $\begin{array}{l}\text { cohort and case- } \\
\text { control type studies }\end{array}$ & not serious & not serious & serious $^{\mathrm{a}}$ & not serious & none & $\begin{array}{c}\oplus \oplus \oplus \bigcirc \\
\text { MODERATE }\end{array}$ \\
\hline \multicolumn{9}{|c|}{ microRNAs not included miR-371a-3p } \\
\hline \multicolumn{2}{|c|}{ Sensitivity } & \multicolumn{2}{|c|}{$0.58(95 \%$ CI: 0.40 to 0.75$)$} & \multicolumn{2}{|c|}{ Specificity } & \multicolumn{3}{|c|}{$0.94(95 \%$ CI: 0.88 to 0.99$)$} \\
\hline $\begin{array}{l}\text { TP } \\
\text { FN }\end{array}$ & $\begin{array}{l}5 \text { studies }[29,35] \\
\text { (596 patients) }\end{array}$ & $\begin{array}{l}\text { cohort and case-control } \\
\text { type studies }\end{array}$ & not serious & not serious & serious $^{\mathrm{b}}$ & not serious & none & $\begin{array}{c}\oplus \oplus \oplus \bigcirc \\
\text { MODERATE }\end{array}$ \\
\hline $\begin{array}{l}\text { TN } \\
\text { FP }\end{array}$ & $\begin{array}{l}5 \text { studies }[29,35] \\
(274 \text { patients })\end{array}$ & $\begin{array}{l}\text { cohort and case-control } \\
\text { type studies }\end{array}$ & not serious & not serious & serious $^{\mathrm{b}}$ & not serious & none & $\begin{array}{c}\oplus \oplus \oplus \bigcirc \\
\text { MODERATE }\end{array}$ \\
\hline \multicolumn{9}{|c|}{ single miR-371a-3p } \\
\hline & ensitivity & 0.84 (95\% CI: 0.76 & 6 to 0.90$)$ & Spec & ficity & 0.95 & $5 \%$ CI: $0.91 \mathrm{t}$ & 0.98) \\
\hline $\begin{array}{l}\text { TP } \\
\text { FN }\end{array}$ & \multicolumn{2}{|c|}{$\begin{array}{c}9 \text { studies [29-35, 37] cohort and case-control } \\
\text { (1183 patients) }\end{array}$} & not serious & not serious & serious $^{\mathrm{c}}$ & not serious & none & $\begin{array}{c}\oplus \oplus \oplus \bigcirc \\
\text { MODERATE }\end{array}$ \\
\hline $\begin{array}{l}\text { TN } \\
\text { FP }\end{array}$ & \multicolumn{2}{|c|}{$\begin{array}{l}9 \text { studies [29-35, 37] cohort and case-control } \\
\text { (669 patients) type studies }\end{array}$} & not serious & not serious & serious $^{\mathrm{c}}$ & not serious & none & $\begin{array}{c}\oplus \oplus \oplus \bigcirc \\
\text { MODERATE }\end{array}$ \\
\hline \multicolumn{9}{|c|}{ multiple microRNAs with miR-371a-3p } \\
\hline \multicolumn{2}{|c|}{ Sensitivity } & \multicolumn{2}{|c|}{$0.95(95 \%$ CI: 0.91 to 1.00$)$} & \multicolumn{2}{|c|}{ Specificity } & \multicolumn{3}{|c|}{$0.73(95 \%$ CI: 0.48 to 0.98$)$} \\
\hline $\begin{array}{l}\text { TP } \\
\text { FN }\end{array}$ & $\begin{array}{c}4 \text { studies } \\
{[35-36,38-39]} \\
\text { (376 patients) }\end{array}$ & $\begin{array}{l}\text { cohort and case-control } \\
\text { type studies }\end{array}$ & not serious & not serious & serious $^{\mathrm{d}}$ & not serious & none & $\begin{array}{c}\oplus \oplus \oplus \bigcirc \\
\text { MODERATE }\end{array}$ \\
\hline $\begin{array}{l}\text { TN } \\
\text { FP }\end{array}$ & $\begin{array}{c}4 \text { studies } \\
{[35-36,38-39]} \\
\text { (182 patients) }\end{array}$ & $\begin{array}{l}\text { cohort and case-control } \\
\text { type studies }\end{array}$ & not serious & not serious & serious $^{\mathrm{d}}$ & not serious & none & $\begin{array}{c}\oplus \oplus \oplus \bigcirc \\
\text { MODERATE }\end{array}$ \\
\hline
\end{tabular}

Table 4 is the original table exported from GRADEpro GDT software.

Bibliography: Morup N 2020 [29], Dieckmann KP 2019 [30], Vilela-SalgueiroB 2018 [31], Radtke A 2017 [32], Pelloni M 2017 [33], Dieckmann KP 2017 [34], Van Agthoven T 2016 [35], Rijlaarsdam MA 2015 [36], Syring I 2015 [37], Gillis AJ 2013 [38], Palmer R.D 2010 [39].

Abbreviations: $\mathrm{Cl}$ : confidence interval; TP: true positive; FN: false negative; TN: true negative; FP: false positive.

Explanations: (a) There were large inconsistencies among the included studies with $R^{2}>50 \%$ and $P<0.1$. (b). There were large inconsistencies among the included studies with $I^{2}>50 \%$ and $P<0.1$. (c) There were large inconsistencies among the included studies with $P^{2}>50 \%$ and $P<0.1$. (d) There were large inconsistencies among the included studies with $P^{2}>50 \%$ and $P<0.1$.

compared with miRNAs. Hence, miRNAs, especially miR-371a-3p, could be promising noninvasive biomarkers of TGCTs. Clinical application tests indicated that miR-371a-3p has an excellent clinical value in the confirmation of TGCTs but has a relatively limited value in the exclusion of TGCTs. miR-371a-3p also exhibited outstanding performance in the generalizability test, especially in the positive test. Therefore, we concluded that miR-371a-3p could be a potentially promising diagnostic marker for TGCT.
To identify factors that influence Sen and Spe, we performed multivariate meta-regression and subgroup analyses. Multivariate meta-regression suggested that miRNA type (miR-371a-3p included or excluded) could be an influencing factor for Sen and miRNA number (single or multiple miRNAs) could be an influencing factor for Spe. However, subgroup analyses showed that Sen could be influenced by differences in specimen, target miRNAs, and number of miRNAs, whereas the Spe could be influenced by the study design. 
Multivariate meta-regressions are more reliable than subgroup analyses because they use a more objective approach to study the effects of covariates on the binary outcome [40]. In addition, among the studies of multiple miRNAs, two with parallel testing showed Spe of 0.91 and 1.00 [35, 39], whereas two with serial testing showed Spe of 0.55 and 0.48 [36, 38]. Thus, different study designs could significantly influence the pooled Spe of multiple miRNAs. Hence, we excluded the type of specimen, the number of miRNAs, and the design of the study as factors that may influence the Sen or Spe. Instead, we concluded that miRNA type (including or excluding miR-371a-3p) could be an important factor influencing Sen.

Despite the contribution of the miRNA type to the heterogeneity of Sen and the higher $I^{2}$ value of Sen and Spe in total miRNA studies (Sen: $93.83 \%$, Spe: 90.2\%) compared to those in single miR-371a-3p studies (Sen: $87.38 \%$, Spe: $54.26 \%$ ), the overall $I^{2}$ value of the diagnostic parameters was relatively high. Hence, the heterogeneity across the selected studies could not be fully explained. This discrepancy could be attributed to three factors. First, there were significant differences between the histological type and clinical stage of patients with TGCTs in the selected studies. Previous studies have noted that patients with the NS subtype (except teratoma) express higher levels of miR-371a-3p than those with the SE subtype, and patients with CS II/III subtypes also express higher levels of miR-371a$3 p$ than those with the CS I subtype [30-31]. Second, our included studies had different threshold levels for the detection of miRNAs. Although the results of Spearman's correlation coefficient and ROC plane showed there was no threshold effect among the studies analyzed, we cannot rule out the possible effects of differences in these cut-off points. Third, the specimen types from which miRNA expression was estimated were different among the selected studies. Previous studies have shown conflicting results regarding the levels of miRNA expression in the serum and tissue of patients with TGCTs. Although Dieckmann et al. [41] did not identify a relationship between the levels of miRNA expression in the serum and tissue of patients with TGCTs, Belge et al. [42] reported a positive correlation between these levels, especially in patients with TGCTs with CS I. Therefore, the influence of the specimen type on heterogeneity remains unclear.

Through this systematic review and meta-analysis, we found that some factors restrict the application of miRNA biomarkers in clinical practice. First, the lack of an appropriate standard cut-off value for miRNA expression might result in heterogeneity among studies [43]. Second, consensus should be reached regarding the best sample type for detection. Since current reports regarding the relationship between the expression level of miRNAs in the serum and tissue are conflicting, further studies are required in this regard. Third, the diagnostic performances of miRNAs for GCNIS and teratoma were not significantly better than those of classic biomarkers. Previous studies revealed that only $51.9 \%$ of patients with GCNIS showed a significant increase in miR-371a-3p levels [33], whereas patients with teratoma showed no significant increase in miR371-373 and miR-302/367 clusters [30]. Several studies have noted this problem, and Lobo et al. reported that the AUC of miR-885-5p used to distinguish mature teratoma from healthy males was 0.89 [44]. Therefore, effective biomarkers are needed to diagnose these two TGCT subtypes [45-46].

This study had several strengths. First, we conducted the first systematic review and meta-analysis, to the best of our knowledge, to evaluate the diagnostic value of miRNAs in TGCTs. Second, we conducted subgroup, meta-regression, and stratified analyses to select the most suitable analysis for clinical application. Our results suggest that multiple miRNAs with miR-371a-3p have similar predictive performances to single miR-371a-3p, and considering that there is no consensus regarding the composition of multiple miRNAs with miR-371a-3p, single miR-371a-3p can be considered a potentially promising diagnostic biomarker for TGCTs in clinical practice. Third, we assessed the quality of the included studies and evidence certainty using the QUADAS-2 and GRADE criteria, respectively. The QUADAS-2 score system suggested that the overall quality of the included studies was high, and the GRADE criteria showed that evidence quality of our results were moderate.

Nonetheless, this study had several limitations. First, the heterogeneity of the selected studies was relatively high. Second, we extracted data from multiple trials within one study, which may increase statistical bias because of the overlapped samples. Third, all populations in the included studies were from Europe, which limits the widespread applicability of the study results.

In brief, our meta-analysis comprehensively explored the diagnostic value of miRNAs in patients with TGCTs. We conclude that miR-371a-3p has a strong diagnostic value for the diagnosis of TGCTs, except teratoma. Therefore, we believed that miR-371a-3p should be considered a potentially promising diagnostic biomarker for TGCTs in clinical practice. However, more prospective studies are needed in future.

\section{CONCLUSIONS}

miR-371a-3p has a high diagnostic value for TGCTs, except teratoma, and should be considered a potentially 
promising diagnostic biomarker for TGCTs in clinical practice.

\section{Abbreviations}

miRNA: MicroRNA; TGCT: Testicular Germ Cell Tumors; AFP: $\alpha$-fetoprotein; Sen: Sensitivity; SE: Seminoma; Spe: Specificity; NMTD: Non-Malignant Testicular Disease; QUADAS-2: Quality Assessment of Diagnostic Accuracy Studies-2; PLR: Positive Likelihood Ratio; NLR: Negative Likelihood Ratio; DOR: Diagnostic Odds Ratio; AUC: Area Under The Curve; CI: Confidence Interval; ROC plane: Receiver Operating Characteristic Space; GCNIS: Germ Cell Neoplasia in situ; NS: Non-Seminoma.

\section{AUTHOR CONTRIBUTIONS}

Conception and design: CZ, XZ; collection and assembly of data: XZ, DL, HL; data analysis and interpretation: $\mathrm{XZ}, \mathrm{CZ}, \mathrm{YG}, \mathrm{RZ}$; manuscript writing: all authors; final approval of manuscript: all authors.

\section{ACKNOWLEDGMENTS}

We gratefully acknowledge the technical assistance provided by the library staff of the Shanghai Jiao Tong University School of Medicine.

\section{CONFLICTS OF INTEREST}

The authors declare no conflicts of interest related to this study.

\section{FUNDING}

This project is supported by Shanghai Pudong New Area Science and Technology Development Fund (PKJ2020-Y12).

\section{REFERENCES}

1. Gilligan T, Lin DW, Aggarwal R, Chism D, Cost N, Derweesh IH, Emamekhoo H, Feldman DR, Geynisman DM, Hancock SL, LaGrange C, Levine EG, Longo T, et al. Testicular Cancer, Version 2.2020, NCCN Clinical Practice Guidelines in Oncology. J Natl Compr Canc Netw. 2019; 17:1529-54.

https://doi.org/10.6004/inccn.2019.0058 PMID:31805523

2. Motzer RJ, Agarwal N, Beard C, Bhayani S, Bolger GB, Buyyounouski MK, Carducci MA, Chang SS, Choueiri TK, Gupta S, Hancock SL, Hudes GR, Jonasch E, et al, and National Comprehensive Cancer Network. Testicular cancer. J Natl Compr Canc Netw. 2012; 10:502-35. https://doi.org/10.6004/incen.2012.0050 PMID:22491049

3. Goldberg K: ASCO 50th anniversary poll names the top 5 advances from the past 50 years, 2014. https://www.asco.org. Accessed 2020.

4. Beyer J, Albers P, Altena R, Aparicio J, Bokemeyer C, Busch J, Cathomas R, Cavallin-Stahl E, Clarke NW, Claßen J, Cohn-Cedermark G, Dahl AA, Daugaard G, et al. Maintaining success, reducing treatment burden, focusing on survivorship: highlights from the third European consensus conference on diagnosis and treatment of germ-cell cancer. Ann Oncol. 2013; 24:878-88.

https://doi.org/10.1093/annonc/mds579 PMID:23152360

5. Gilligan TD, Seidenfeld J, Basch EM, Einhorn LH, Fancher T, Smith DC, Stephenson AJ, Vaughn DJ, Cosby R, Hayes DF, and American Society of Clinical Oncology. American Society of Clinical Oncology Clinical Practice Guideline on uses of serum tumor markers in adult males with germ cell tumors. J Clin Oncol. 2010; 28:3388-404.

https://doi.org/10.1200/JCO.2009.26.4481

PMID:20530278

6. Albers P, Albrecht W, Algaba F, Bokemeyer C, CohnCedermark G, Fizazi K, Horwich A, Laguna MP, Nicolai $\mathrm{N}$, Oldenburg J, and European Association of Urology. Guidelines on Testicular Cancer: 2015 Update. Eur Urol. 2015; 68:1054-68.

https://doi.org/10.1016/j.eururo.2015.07.044 PMID:26297604

7. Dieckmann KP, Richter-Simonsen $H$, Kulejewski $M$, Ikogho R, Zecha H, Anheuser P, Pichlmeier U, Isbarn H. Testicular Germ-Cell Tumours: A Descriptive Analysis of Clinical Characteristics at First Presentation. Urol Int. 2018; 100:409-19.

https://doi.org/10.1159/000488284

PMID:29649815

8. von Eyben FE. Laboratory markers and germ cell tumors. Crit Rev Clin Lab Sci. 2003; 40:377-427. https://doi.org/10.1080/10408360390247814 PMID:14582602

9. Peng $Y$, Croce $\mathrm{CM}$. The role of MicroRNAs in human cancer. Signal Transduct Target Ther. 2016; 1:15004. https://doi.org/10.1038/sigtrans.2015.4 PMID:29263891

10. Belge G, Dieckmann KP, Spiekermann M, Balks T, Bullerdiek J. Serum levels of microRNAs miR-371-3: a novel class of serum biomarkers for testicular germ cell tumors? Eur Urol. 2012; 61:1068-69. https://doi.org/10.1016/i.eururo.2012.02.037 PMID:22386195 
11. van Agthoven $\mathrm{T}$, Eijkenboom WMH, Looijenga LHJ. microRNA-371a-3p as informative biomarker for the follow-up of testicular germ cell cancer patients. Cell Oncol (Dordr). 2017; 40:379-88.

https://doi.org/10.1007/s13402-017-0333-9

PMID:28612337

12. Radtke A, Hennig F, Ikogho R, Hammel J, Anheuser P, Wülfing C, Belge G, Dieckmann KP. The Novel Biomarker of Germ Cell Tumours, Micro-RNA-371a$3 p$, Has a Very Rapid Decay in Patients with Clinical Stage 1. Urol Int. 2018; 100:470-75. https://doi.org/10.1159/000488771 PMID:29698973

13. Moher D, Liberati A, Tetzlaff J, Altman DG, and PRISMA Group. Preferred reporting items for systematic reviews and meta-analyses: the PRISMA statement. BMJ. 2009; 339:b2535.

https://doi.org/10.1136/bmj.b2535

PMID:19622551

14. Whiting PF, Rutjes AW, Westwood ME, Mallett S, Deeks JJ, Reitsma JB, Leeflang MM, Sterne JA, Bossuyt PM, and QUADAS-2 Group. QUADAS-2: a revised tool for the quality assessment of diagnostic accuracy studies. Ann Intern Med. 2011; 155:529-36. https://doi.org/10.7326/0003-4819-155-8201110180-00009 PMID:22007046

15. Reitsma JB, Glas AS, Rutjes AW, Scholten RJ, Bossuyt PM, Zwinderman AH. Bivariate analysis of sensitivity and specificity produces informative summary measures in diagnostic reviews. J Clin Epidemiol. 2005; 58:982-90.

https://doi.org/10.1016/j.jclinepi.2005.02.022 PMID:16168343

16. Higgins JP, Thompson SG, Deeks JJ, Altman DG. Measuring inconsistency in meta-analyses. BMJ. 2003; 327:557-60. https://doi.org/10.1136/bmi.327.7414.557 PMID: 12958120

17. Dwamena B. MIDAS: Statamodule for meta-analytical integration of diagnostic accuracy studies. 2007. https://econpapers.repec.org. Accessed 2020.

18. Zamora J, Abraira V, Muriel A, Khan K, Coomarasamy A. Meta-DiSc: a software for meta-analysis of test accuracy data. BMC Med Res Methodol. 2006; 6:31. https://doi.org/10.1186/1471-2288-6-31 PMID:16836745

19. Moses LE, Shapiro D, Littenberg B. Combining independent studies of a diagnostic test into a summary ROC curve: data-analytic approaches and some additional considerations. Stat Med. 1993; $12: 1293-316$ https://doi.org/10.1002/sim.4780121403 PMID:8210827

20. Dinnes J, Deeks J, Kirby J, Roderick P. A methodological review of how heterogeneity has been examined in systematic reviews of diagnostic test accuracy. Health Technol Assess. 2005; 9:1-113, iii. https://doi.org/10.3310/hta9120 PMID:15774235

21. Small DS. Sensitivity analysis for instrumental variables regression with overidentifying restrictions. J Am Stat Assoc. 2007; 102:1049-58.

22. Begg CB, Berlin JA. Publication bias and dissemination of clinical research. J Natl Cancer Inst. 1989; 81:107-15.

https://doi.org/10.1093/jnci/81.2.107

PMID:2642556

23. Deeks JJ, Macaskill P, Irwig L. The performance of tests of publication bias and other sample size effects in systematic reviews of diagnostic test accuracy was assessed. J Clin Epidemiol. 2005; 58:882-93.

https://doi.org/10.1016/j.jclinepi.2005.01.016 PMID:16085191

24. Rubinstein ML, Kraft CS, Parrott JS. Determining qualitative effect size ratings using a likelihood ratio scatter matrix in diagnostic test accuracy systematic reviews. Diagnosis (Berl). 2018; 5:205-14.

https://doi.org/10.1515/dx-2018-0061

PMID: $\underline{30243015}$

25. Fagan TJ. Letter: Nomogram for Bayes theorem. N Engl J Med. 1975; 293:257. https://doi.org/10.1056/NEJM197507312930513 PMID:1143310

26. Jaeschke R, Guyatt GH, Sackett DL. Users' guides to the medical literature. III. How to use an article about a diagnostic test. B. What are the results and will they help me in caring for my patients? The Evidence-Based Medicine Working Group. JAMA. 1994; 271:703-07. PMID:8309035

27. GRADEpro GDT: GRADEpro Guideline Development Tool [Software]. McMaster University, 2020 (developed by Evidence Prime, Inc.).

28. Schünemann H, Brożek J, Guyatt G, Oxman A, editors. GRADE handbook for grading quality of evidence and strength of recommendations. Updated October 2013. The GRADE Working Group, 2013.

29. Mørup N, Rajpert-De Meyts E, Juul A, Daugaard G, Almstrup K. Evaluation of Circulating miRNA Biomarkers of Testicular Germ Cell Tumors during Therapy and Follow-up-A Copenhagen Experience. Cancers (Basel). 2020; 12:759. 
https://doi.org/10.3390/cancers12030759 PMID:32210101

30. Dieckmann KP, Radtke A, Geczi L, Matthies C, Anheuser P, Eckardt U, Sommer J, Zengerling F, Trenti E, Pichler R, Belz H, Zastrow S, Winter A, et al. Serum Levels of MicroRNA-371a-3p (M371 Test) as a New Biomarker of Testicular Germ Cell Tumors: Results of a Prospective Multicentric Study. J Clin Oncol. 2019; 37:1412-23.

https://doi.org/10.1200/JCO.18.01480

PMID: $\underline{30875280}$

31. Vilela-Salgueiro B, Barros-Silva D, Lobo J, Costa AL, Guimarães R, Cantante M, Lopes P, Braga I, Oliveira J, Henrique R, Jerónimo C. Germ cell tumour subtypes display differential expression of microRNA371a-3p. Philos Trans R Soc Lond B Biol Sci. 2018; 373:20170338. https://doi.org/10.1098/rstb.2017.0338 PMID:29685967

32. Radtke A, Cremers JF, Kliesch S, Riek S, Junker K, Mohamed SA, Anheuser P, Belge G, Dieckmann KP. Can germ cell neoplasia in situ be diagnosed by measuring serum levels of microRNA371a-3p? J Cancer Res Clin Oncol. 2017; 143:2383-92. https://doi.org/10.1007/s00432-017-2490-7 PMID:28819887

33. Pelloni M, Coltrinari G, Paoli D, Pallotti F, Lombardo F, Lenzi A, Gandini L. Differential expression of miRNAs in the seminal plasma and serum of testicular cancer patients. Endocrine. 2017; 57:518-27. https://doi.org/10.1007/s12020-016-1150-z PMID:27796811

34. Dieckmann KP, Radtke A, Spiekermann M, Balks T, Matthies C, Becker P, Ruf C, Oing C, Oechsle K, Bokemeyer C, Hammel J, Melchior S, Wosniok W, Belge G. Corrigendum re: "Serum Levels of MicroRNA miR-371a-3p: A Sensitive and Specific New Biomarker for Germ Cell Tumours" [Eur Urol. 2017; 71:213-20]. Eur Urol. 2017; 71:e161.

https://doi.org/10.1016/i.eururo.2017.01.041 PMID:28209431

35. van Agthoven T, Looijenga LHJ. Accurate primary germ cell cancer diagnosis using serum based microRNA detection (ampTSmiR test). Oncotarget. 2016; 8:58037-49.

https://doi.org/10.18632/oncotarget.10867 PMID:28938535

36. Rijlaarsdam MA, van Agthoven $T$, Gillis AJ, Patel $S$, Hayashibara K, Lee KY, Looijenga LH. Identification of known and novel germ cell cancer-specific (embryonic) miRs in serum by high-throughput profiling. Andrology. 2015; 3:85-91. https://doi.org/10.1111/andr.298 PMID:25382245
37. Syring I, Bartels J, Holdenrieder S, Kristiansen G, Müller SC, Ellinger J. Circulating serum miRNA (miR367-3p, miR-371a-3p, miR-372-3p and miR-373-3p) as biomarkers in patients with testicular germ cell cancer. J Urol. 2015; 193:331-37.

https://doi.org/10.1016/i.juro.2014.07.010 PMID:25046619

38. Gillis AJ, Rijlaarsdam MA, Eini R, Dorssers LC, Biermann K, Murray MJ, Nicholson JC, Coleman N, Dieckmann KP, Belge G, Bullerdiek J, Xu T, Bernard N, Looijenga LH. Targeted serum miRNA (TSmiR) test for diagnosis and follow-up of (testicular) germ cell cancer patients: a proof of principle. Mol Oncol. 2013; 7:1083-92.

https://doi.org/10.1016/i.molonc.2013.08.002 PMID:24012110

39. Palmer RD, Murray MJ, Saini HK, van Dongen S, AbreuGoodger C, Muralidhar B, Pett MR, Thornton CM, Nicholson JC, Enright AJ, Coleman N, and Childrens Cancer and Leukaemia Group. Malignant germ cell tumors display common microRNA profiles resulting in global changes in expression of messenger RNA targets. Cancer Res. 2010; 70:2911-23. https://doi.org/10.1158/0008-5472.CAN-09-3301 PMID:20332240

40. Wang H, Peng J, Wang B, Lu X, Zheng JZ, Wang K, Tu $\mathrm{XM}$, Feng C. Inconsistency Between Univariate and Multiple Logistic Regressions. Shanghai Arch Psychiatry. 2017; 29:124-28.

PMID:28765686

41. Dieckmann KP, Spiekermann M, Balks T, Flor I, Löning T, Bullerdiek J, Belge G. MicroRNAs miR-371-3 in serum as diagnostic tools in the management of testicular germ cell tumours. $\mathrm{Br} J$ Cancer. 2012; 107:1754-60.

https://doi.org/10.1038/bjc.2012.469

PMID:23059743

42. Belge G, Hennig F, Dumlupinar C, Grobelny F, Junker K, Radtke A, Dieckmann KP. Graded expression of microRNA-371a-3p in tumor tissues, contralateral testes, and in serum of patients with testicular germ cell tumor. Oncotarget. 2020; 11:1462-73.

https://doi.org/10.18632/oncotarget.27565 PMID: $\underline{32363003}$

43. Holländer N, Sauerbrei W, Schumacher M. Confidence intervals for the effect of a prognostic factor after selection of an 'optimal' cutpoint. Stat Med. 2004; 23:1701-13.

https://doi.org/10.1002/sim.1611 PMID:15160403

44. Lobo J, Gillis AJM, van den Berg A, Dorssers LCJ, Belge G, Dieckmann KP, Roest HP, van der Laan LW, Gietema J, Hamilton RJ, Jerónimo C, Henrique R, 
Salvatori D, Looijenga LHJ. Identification and Validation Model for Informative Liquid Biopsy-Based microRNA Biomarkers: Insights from Germ Cell Tumor In Vitro, In Vivo and Patient-Derived Data. Cells. 2019; 8:1637.

https://doi.org/10.3390/cells8121637

PMID:31847394

45. Almstrup K, Lobo J, Mørup N, Belge G, Rajpert-De Meyts E, Looijenga LHJ, Dieckmann KP. Application of miRNAs in the diagnosis and monitoring of testicular germ cell tumours. Nat Rev Urol. 2020; 17:201-13. https://doi.org/10.1038/s41585-020-0296-x

PMID: 32157202

46. Batool A, Karimi N, Wu XN, Chen SR, Liu YX. Testicular germ cell tumor: a comprehensive review. Cell Mol Life Sci. 2019; 76:1713-27.

https://doi.org/10.1007/s00018-019-03022-7

PMID: $\underline{30671589}$ 


\section{SUPPLEMENTARY MATERIALS}

\section{Supplementary Figures}
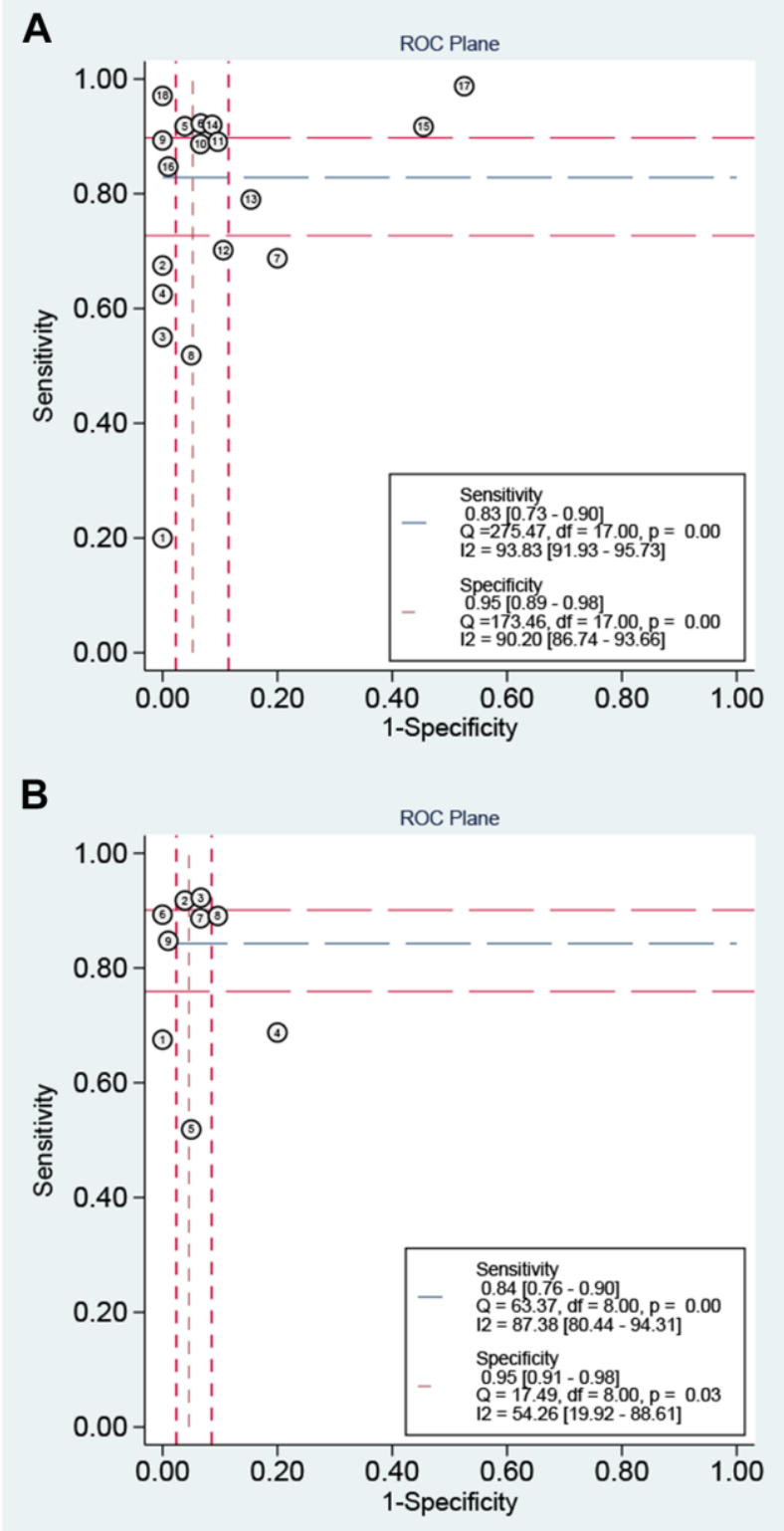

Supplementary Figure 1. ROC plane of the included studies. (A) ROC plane of total microRNAs; (B) ROC plane of single miR-371-3p. 
Sensitivity analysis of sensitivity

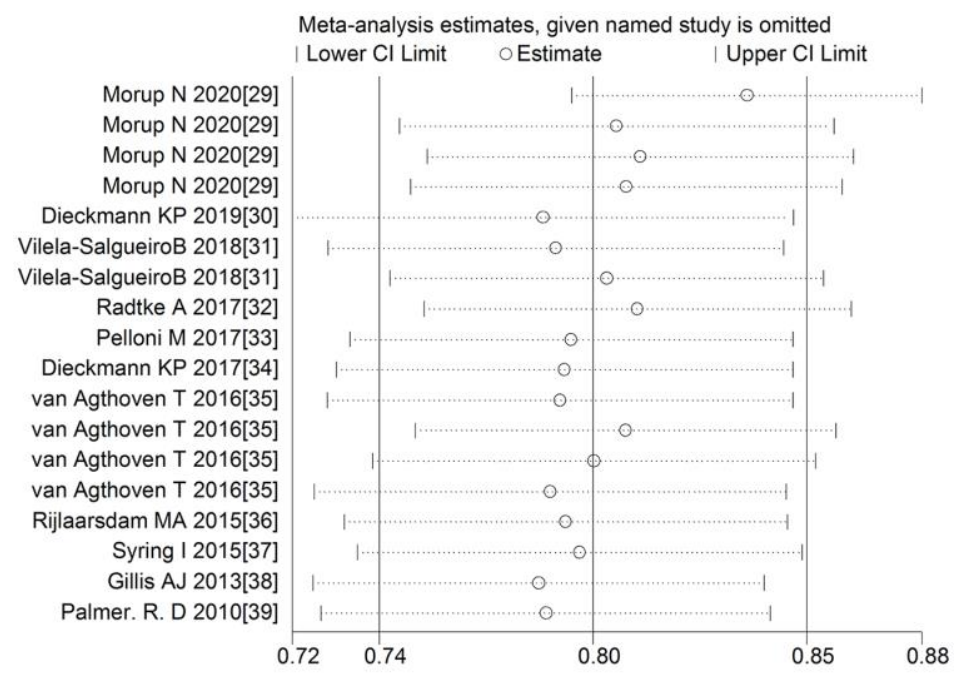

\section{Sensitivity analysis of specificity}

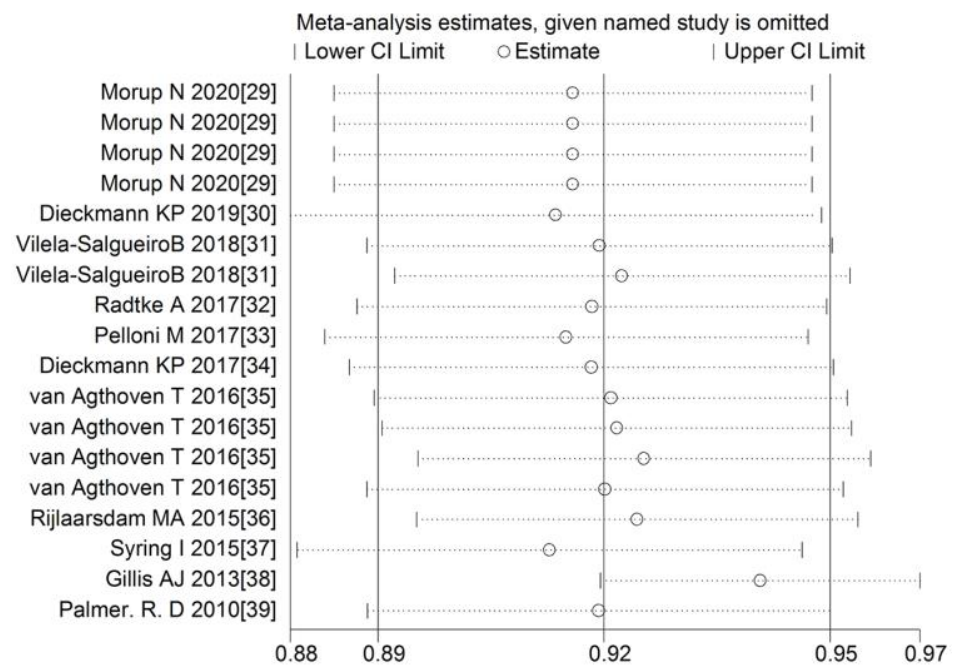

Supplementary Figure 2. Sensitivity analysis of the included studies. 


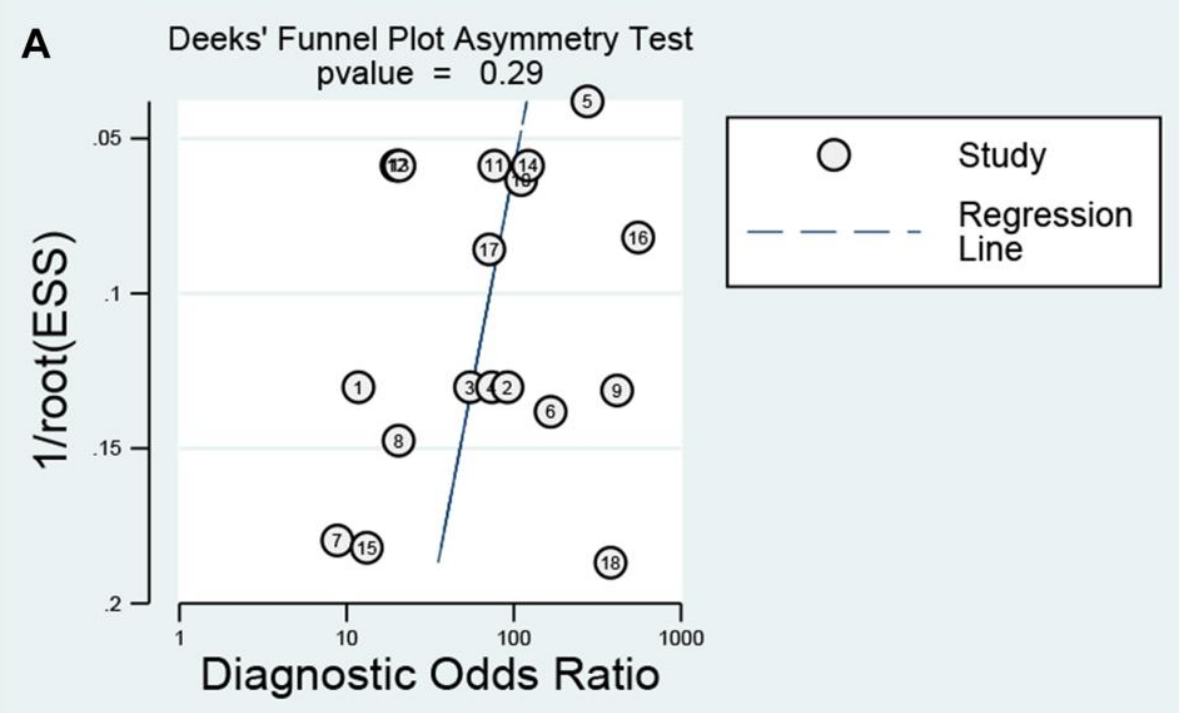

B

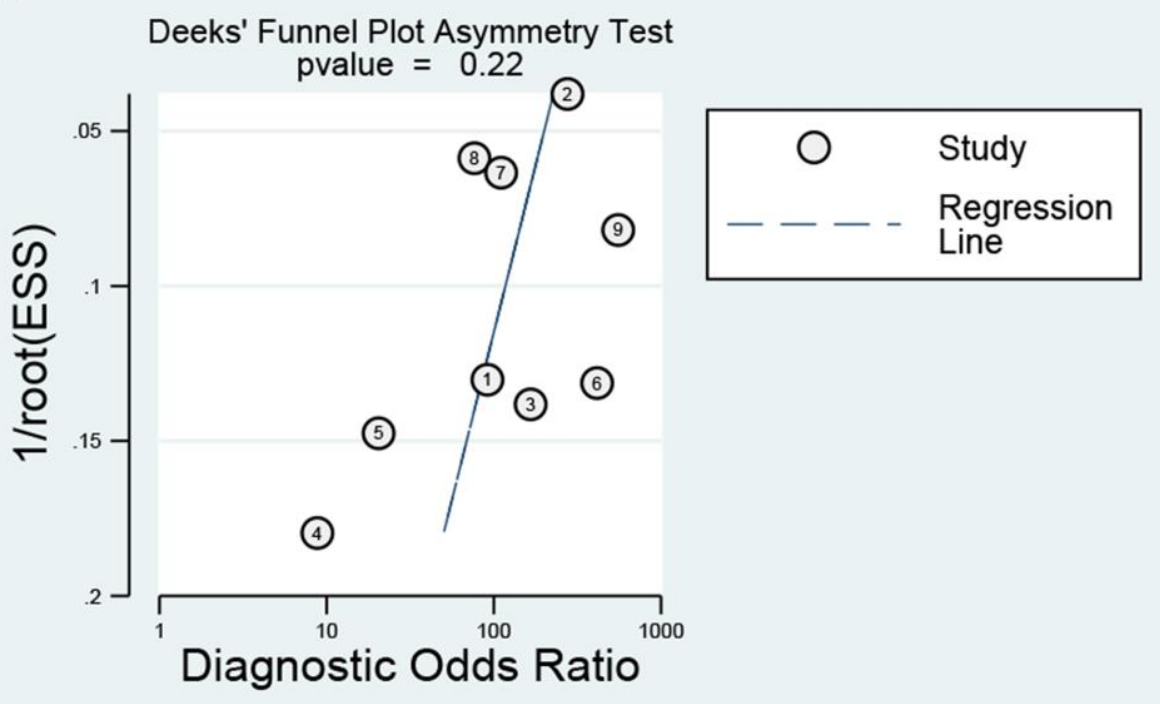

Supplementary Figure 3. Estimation of the publication bias by Deeks' funnel plots. (A) Deeks' funnel plots of total microRNAs $(P=0.29)$; (B) Deeks' funnel plots of single miR-371a-3p $(P=0.22)$. 
A

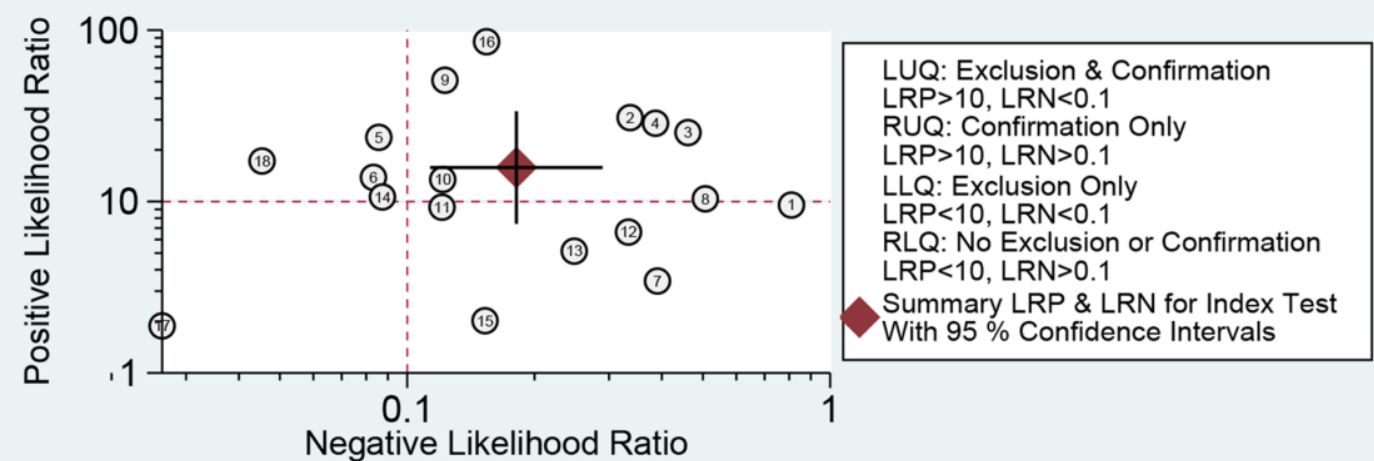

B

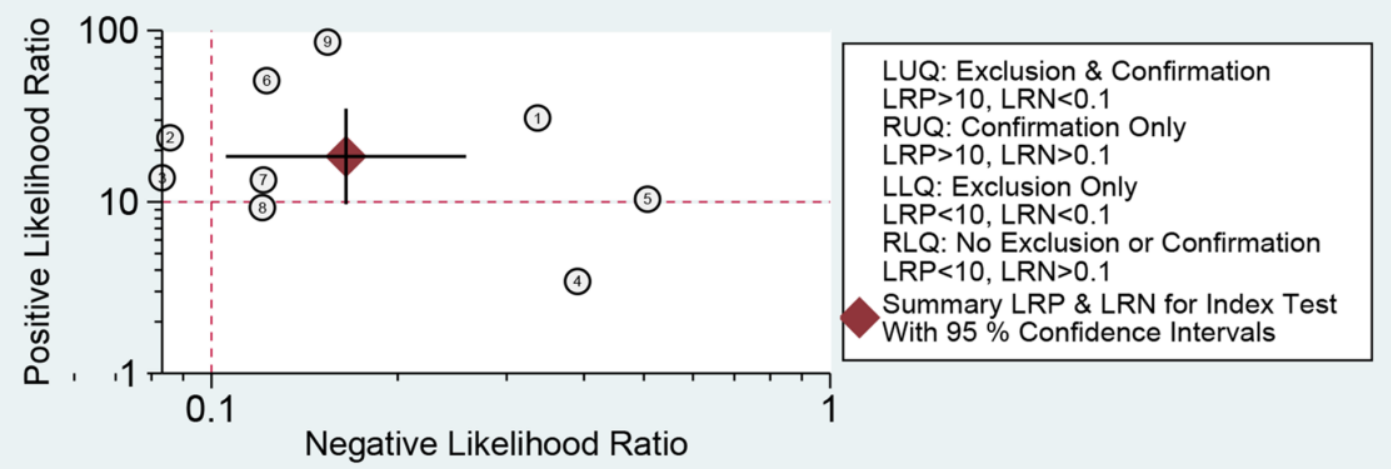

Supplementary Figure 4. Likelihood ratio scattergram. (A) Likelihood ratio scattergram of total microRNAs; (B) Likelihood ratio scattergram of single miR-371a-3p. Abbreviations: LRP: positive likelihood ratio; LRN: negative likelihood ratio; LUQ: left upper quadrant; RUQ: right upper quadrant; LLQ: left lower quadrant; RLQ: right lower quadrant. 


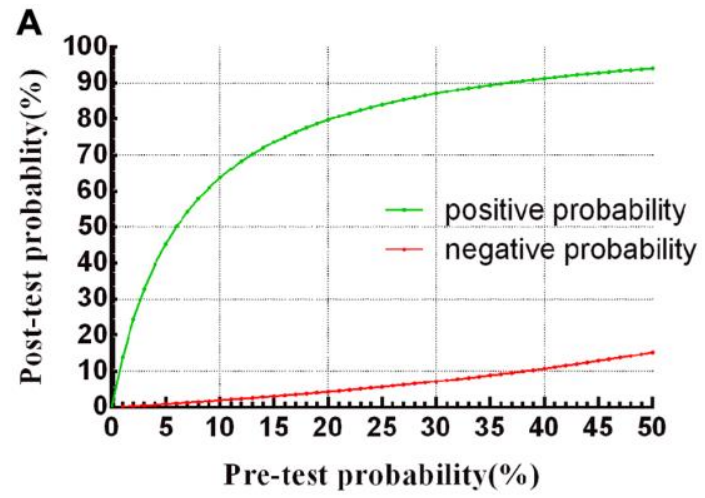

C

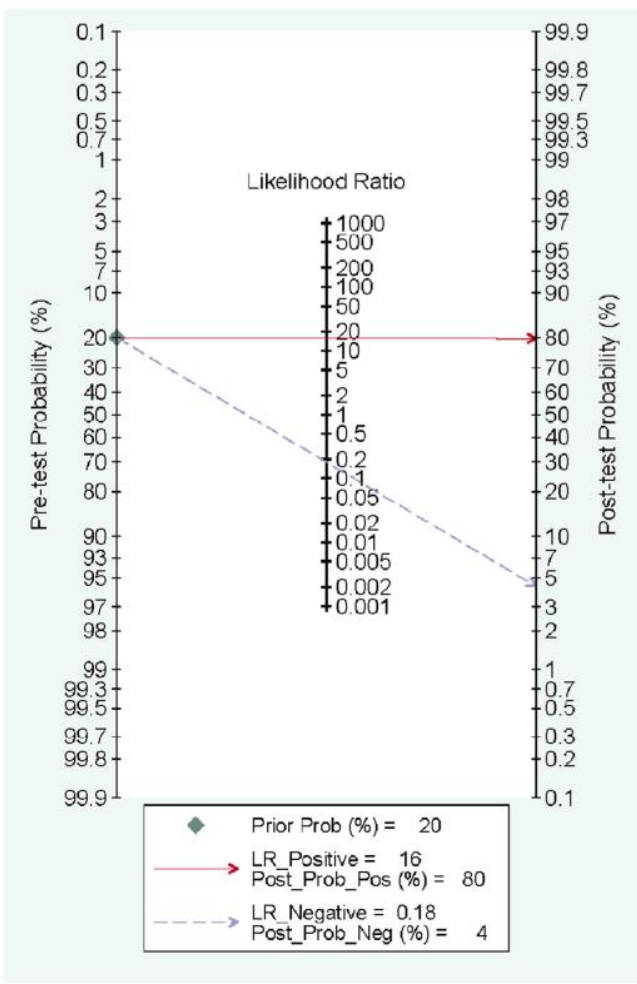

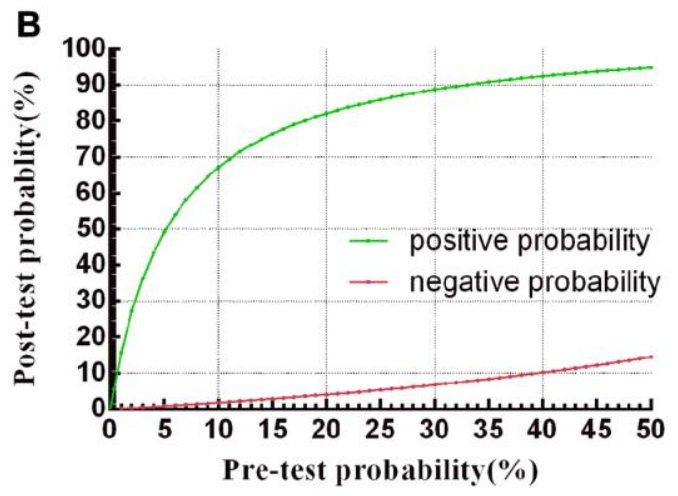

D

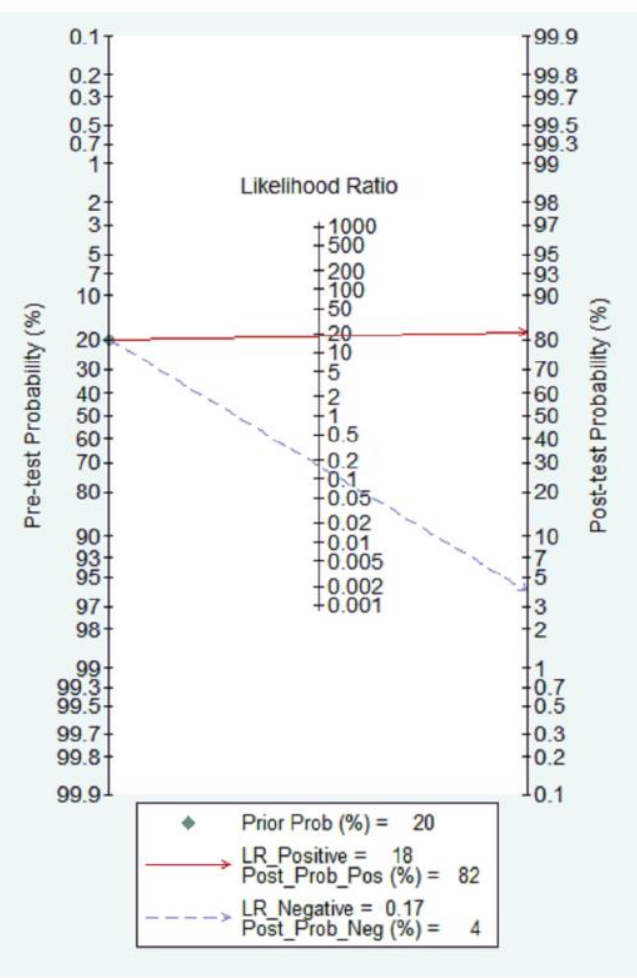

Supplementary Figure 5. Line charts and Fagan's plots indicating diagnostic probability. (A) diagnostic probability line chart of total microRNAs; (B) diagnostic probability line chart of single miR-371a-3p; (C) Fagan's plot based on the TGCT pre-test probability of $20 \%$ for total microRNAs; (D) Fagan's plot based on the TGCT pre-test probability of 20\% for single miR-371a-3p. 


\section{Supplementary Tables}

Supplementary Table 1. PubMed search strategy.

Recent queries in Pubmed

\begin{tabular}{|c|c|c|}
\hline Search & Query & Items found \\
\hline \#10 & $\begin{array}{l}\text { Search ((((((Testicular Germ Cell Tumors[Title/Abstract]) OR Testicular Germ Cell } \\
\text { Tumor[Title/Abstract])) OR ((“Germinoma”[Mesh]) OR Germinomas)) OR ((“Testicular } \\
\text { Neoplasms”[Mesh]) OR ((Neoplasm, Testicular[Title/Abstract]) OR Testicular } \\
\text { Neoplasm[Title/Abstract]) OR Testicular Tumors[Title/Abstract]) OR Testis } \\
\text { Neoplasms[Title/Abstract]) OR Neoplasms, Testicular[Title/Abstract]) OR Neoplasms, } \\
\text { Testis[Title/Abstract]) OR Neoplasm, Testis[Title/Abstract]) OR Testis Neoplasm[Title/Abstract]) } \\
\text { OR Tumor of Rete Testis[Title/Abstract]) OR Rete Testis Tumor[Title/Abstract]) OR Rete Testis } \\
\text { Tumors[Title/Abstract]) OR Testis Tumor, Rete[Title/Abstract]) OR Testis Tumors, } \\
\text { Rete[Title/Abstract]) OR Cancer of Testis[Title/Abstract]) OR Testis Cancers[Title/Abstract]) OR } \\
\text { Testis Cancer[Title/Abstract]) OR Cancer, Testis[Title/Abstract]) OR Cancers, } \\
\text { Testis[Title/Abstract]) OR Cancer of the Testes[Title/Abstract]) OR Cancer of the } \\
\text { Testis[Title/Abstract]) OR Testicular Cancer[Title/Abstract]) OR Cancer, } \\
\text { Testicular[Title/Abstract]) OR Cancers, Testicular[Title/Abstract]) OR Testicular } \\
\text { Cancers[Title/Abstract])))) AND ((“MicroRNAs”[Mesh]) OR } \\
\text { ((((((((((((((((MicroRNA[Title/Abstract]) OR miRNAs[Title/Abstract]) OR Micro } \\
\text { RNA[Title/Abstract]) OR RNA, Micro[Title/Abstract]) OR miRNA[Title/Abstract]) OR Primary } \\
\text { MicroRNA[Title/Abstract]) OR MicroRNA, Primary[Title/Abstract]) OR Primary } \\
\text { miRNA[Title/Abstract]) OR miRNA, Primary[Title/Abstract]) OR pri-miRNA[Title/Abstract]) OR } \\
\text { pri miRNA[Title/Abstract]) OR RNA, Small Temporal[Title/Abstract]) OR Temporal RNA, } \\
\text { Small[Title/Abstract]) OR stRNA[Title/Abstract]) OR Small Temporal RNA[Title/Abstract]) OR } \\
\text { pre-miRNA[Title/Abstract]) OR pre miRNA[Title/Abstract])) }\end{array}$ & 226 \\
\hline \#9 & $\begin{array}{l}\text { Search ((((Testicular Germ Cell Tumors[Title/Abstract]) OR Testicular Germ Cell } \\
\text { Tumor[Title/Abstract])) OR ((“Germinoma”[Mesh]) OR Germinomas)) OR ((“Testicular } \\
\text { Neoplasms”[Mesh]) OR ((Neoplasm, Testicular[Title/Abstract]) OR Testicular } \\
\text { Neoplasm[Title/Abstract]) OR Testicular Tumors[Title/Abstract]) OR Testis } \\
\text { Neoplasms[Title/Abstract]) OR Neoplasms, Testicular[Title/Abstract]) OR Neoplasms, } \\
\text { Testis[Title/Abstract]) OR Neoplasm, Testis[Title/Abstract]) OR Testis Neoplasm[Title/Abstract]) } \\
\text { OR Tumor of Rete Testis[Title/Abstract]) OR Rete Testis Tumor[Title/Abstract]) OR Rete Testis } \\
\text { Tumors[Title/Abstract]) OR Testis Tumor, Rete[Title/Abstract]) OR Testis Tumors, } \\
\text { Rete[Title/Abstract]) OR Cancer of Testis[Title/Abstract]) OR Testis Cancers[Title/Abstract]) OR } \\
\text { Testis Cancer[Title/Abstract]) OR Cancer, Testis[Title/Abstract]) OR Cancers, } \\
\text { Testis[Title/Abstract]) OR Cancer of the Testes[Title/Abstract]) OR Cancer of the } \\
\text { Testis[Title/Abstract]) OR Testicular Cancer[Title/Abstract]) OR Cancer, } \\
\text { Testicular[Title/Abstract]) OR Cancers, Testicular[Title/Abstract]) OR Testicular } \\
\text { Cancers[Title/Abstract])) }\end{array}$ & 38313 \\
\hline \#8 & $\begin{array}{l}\text { Search (Testicular Germ Cell Tumors[Title/Abstract]) OR Testicular Germ Cell } \\
\text { Tumor[Title/Abstract] }\end{array}$ & 1981 \\
\hline \#7 & Search (“Germinoma”[Mesh]) OR Germinomas[Title/Abstract] & 11464 \\
\hline \#6 & $\begin{array}{l}\text { Search (“Testicular Neoplasms”[Mesh]) OR ((Neoplasm, Testicular[Title/Abstract]) OR Testicular } \\
\text { Neoplasm[Title/Abstract]) OR Testicular Tumors[Title/Abstract]) OR Testis } \\
\text { Neoplasms[Title/Abstract]) OR Neoplasms, Testicular[Title/Abstract]) OR Neoplasms, } \\
\text { Testis[Title/Abstract]) OR Neoplasm, Testis[Title/Abstract]) OR Testis Neoplasm[Title/Abstract]) } \\
\text { OR Tumor of Rete Testis[Title/Abstract]) OR Rete Testis Tumor[Title/Abstract]) OR Rete Testis } \\
\text { Tumors[Title/Abstract]) OR Testis Tumor, Rete[Title/Abstract]) OR Testis Tumors, } \\
\text { Rete[Title/Abstract]) OR Cancer of Testis[Title/Abstract]) OR Testis Cancers[Title/Abstract]) OR } \\
\text { Testis Cancer[Title/Abstract]) OR Cancer, Testis[Title/Abstract]) OR Cancers, } \\
\text { Testis[Title/Abstract]) OR Cancer of the Testes[Title/Abstract]) OR Cancer of the } \\
\text { Testis[Title/Abstract]) OR Testicular Cancer[Title/Abstract]) OR Cancer, } \\
\text { Testicular[Title/Abstract]) OR Cancers, Testicular[Title/Abstract]) OR Testicular } \\
\text { Cancers[Title/Abstract]) }\end{array}$ & 33543 \\
\hline
\end{tabular}


Testis[Title/Abstract]) OR Testis Neoplasm[Title/Abstract]) OR Tumor of Rete

Testis[Title/Abstract]) OR Rete Testis Tumor[Title/Abstract]) OR Rete Testis

Tumors[Title/Abstract]) OR Testis Tumor, Rete[Title/Abstract]) OR Testis Tumors,

Rete[Title/Abstract]) OR Cancer of Testis[Title/Abstract]) OR Testis Cancers[Title/Abstract]) OR

Testis Cancer[Title/Abstract]) OR Cancer, Testis[Title/Abstract]) OR Cancers,

Testis[Title/Abstract]) OR Cancer of the Testes[Title/Abstract]) OR Cancer of the

Testis[Title/Abstract]) OR Testicular Cancer[Title/Abstract]) OR Cancer,

Testicular[Title/Abstract]) OR Cancers, Testicular[Title/Abstract]) OR Testicular

Cancers[Title/Abstract]

\#4 Search "Testicular Neoplasms”[Mesh]

\#3 Search $(((()((()((()(()($ MicroRNA[Title/Abstract]) OR miRNAs[Title/Abstract]) OR Micro

RNA[Title/Abstract]) OR RNA, Micro[Title/Abstract]) OR miRNA[Title/Abstract]) OR Primary

MicroRNA[Title/Abstract]) OR MicroRNA, Primary[Title/Abstract]) OR Primary

miRNA[Title/Abstract]) OR miRNA, Primary[Title/Abstract]) OR pri-miRNA[Title/Abstract]) OR

pri miRNA[Title/Abstract]) OR RNA, Small Temporal[Title/Abstract]) OR Temporal RNA,

Small[Title/Abstract]) OR stRNA[Title/Abstract]) OR Small Temporal RNA[Title/Abstract]) OR pre-miRNA[Title/Abstract]) OR pre miRNA[Title/Abstract])) OR “MicroRNAs”[Mesh]

RNA[Title/Abstract]) OR RNA, Micro[Title/Abstract]) OR miRNA[Title/Abstract]) OR Primary MicroRNA[Title/Abstract]) OR MicroRNA, Primary[Title/Abstract]) OR Primary miRNA[Title/Abstract]) OR miRNA, Primary[Title/Abstract]) OR pri-miRNA[Title/Abstract]) OR pri miRNA[Title/Abstract]) OR RNA, Small Temporal[Title/Abstract]) OR Temporal RNA, Small[Title/Abstract]) OR stRNA[Title/Abstract]) OR Small Temporal RNA[Title/Abstract]) OR pre-miRNA[Title/Abstract]) OR pre miRNA[Title/Abstract]

Supplementary Table 2. Meta-regression of sensitivity and specificity.

\begin{tabular}{|c|c|c|c|c|}
\hline \multirow{2}{*}{ Independent variable } & Sensitivity & \multirow{2}{*}{$P$} & Specificity & \multirow{2}{*}{$\boldsymbol{P}$} \\
\hline & $(95 \% \mathrm{CI})$ & & $(95 \% \mathrm{CI})$ & \\
\hline Design type & $-0.08(-0.31,0.15)$ & 0.455 & $0.13(-0.02,0.29)$ & 0.075 \\
\hline Specimen type & $0.00(-0.22,0.22)$ & 0.993 & $0.05(-0.12,0.23)$ & 0.514 \\
\hline Controls & $-0.09(-0.28,0.10)$ & 0.333 & $-0.11(-0.25,0.03)$ & 0.106 \\
\hline miRNA type & $-0.23(-0.44,-0.02)$ & 0.035 & $-0.07(-0.20,0.07)$ & 0.289 \\
\hline miRNA number & $0.83(-0.11,0.28)$ & 0.383 & $-0.20(-0.35,-0.05)$ & 0.015 \\
\hline
\end{tabular}

